\title{
An Intelligent Clinical Decision Support System for Patient-Specific Predictions to Improve Cervical Intraepithelial Neoplasia Detection
}

\author{
Panagiotis Bountris, ${ }^{1}$ Maria Haritou, ${ }^{2}$ Abraham Pouliakis, ${ }^{3}$ Niki Margari, ${ }^{3}$ \\ Maria Kyrgiou, ${ }^{4,5}$ Aris Spathis, ${ }^{3}$ Asimakis Pappas, ${ }^{6}$ Ioannis Panayiotides, ${ }^{7}$ \\ Evangelos A. Paraskevaidis, ${ }^{8}$ Petros Karakitsos, ${ }^{3}$ and Dimitrios-Dionyssios Koutsouris ${ }^{1}$ \\ ${ }^{1}$ Biomedical Engineering Laboratory, School of Electrical and Computer Engineering, National Technical University of Athens, \\ Iroon Politechniou 9, 15773 Zografou Campus, Athens, Greece \\ ${ }^{2}$ Institute of Communication and Computer Systems, National Technical University of Athens, Iroon Politechniou 9, \\ 15773 Zografou Campus, Athens, Greece \\ ${ }^{3}$ Department of Cytopathology, School of Medicine, University General Hospital “ATTIKON”, University of Athens, Rimini 1, \\ 12462 Athens, Greece \\ ${ }^{4}$ West London Gynaecological Cancer Center, Queen Charlotte's and Chelsea, Hammersmith Hospital, Imperial Healthcare NHS Trust, \\ London W12 OHS, UK \\ ${ }^{5}$ Division of Surgery and Cancer, Faculty of Medicine, Imperial College, London W12 0NN, UK \\ ${ }^{6} 3 \mathrm{rd}$ Department of Obstetrics and Gynecology, University General Hospital "ATTIKON", School of Medicine, University of Athens, \\ Rimini 1, 12462 Athens, Greece \\ ${ }^{7}$ 2nd Department of Pathology, University General Hospital "ATTIKON”, School of Medicine, University of Athens, Rimini 1, \\ 12462 Athens, Greece \\ ${ }^{8}$ Department of Obstetrics and Gynecology, University Hospital of Ioannina, St. Niarchou Str, 45500 Ioannina, Greece
}

Correspondence should be addressed to Panagiotis Bountris; pbountris@biomed.ntua.gr

Received 9 October 2013; Revised 10 February 2014; Accepted 16 March 2014; Published 9 April 2014

Academic Editor: Nasimul Noman

Copyright ( 2014 Panagiotis Bountris et al. This is an open access article distributed under the Creative Commons Attribution License, which permits unrestricted use, distribution, and reproduction in any medium, provided the original work is properly cited.

Nowadays, there are molecular biology techniques providing information related to cervical cancer and its cause: the human Papillomavirus (HPV), including DNA microarrays identifying HPV subtypes, mRNA techniques such as nucleic acid based amplification or flow cytometry identifying E6/E7 oncogenes, and immunocytochemistry techniques such as overexpression of p16. Each one of these techniques has its own performance, limitations and advantages, thus a combinatorial approach via computational intelligence methods could exploit the benefits of each method and produce more accurate results. In this article we propose a clinical decision support system (CDSS), composed by artificial neural networks, intelligently combining the results of classic and ancillary techniques for diagnostic accuracy improvement. We evaluated this method on 740 cases with complete series of cytological assessment, molecular tests, and colposcopy examination. The CDSS demonstrated high sensitivity (89.4\%), high specificity (97.1\%), high positive predictive value (89.4\%), and high negative predictive value (97.1\%), for detecting cervical intraepithelial neoplasia grade 2 or worse $(\mathrm{CIN} 2+)$. In comparison to the tests involved in this study and their combinations, the CDSS produced the most balanced results in terms of sensitivity, specificity, PPV, and NPV. The proposed system may reduce the referral rate for colposcopy and guide personalised management and therapeutic interventions.

\section{Introduction}

Cervical cancer is the third most common cancer and the fourth leading cause of cancer death in females worldwide
[1]. Cervical cancer is known to be caused almost always by human papillomavirus (HPV) infection which is the commonest sexually transmitted infection worldwide. However, the presence of HPV does not always lead to disease [2]. 
About 100 types of HPV virus have been identified that can infect humans. Among them, at least 15 are oncogenic and thus can cause cancer of the cervix [3,4]. Improved understanding of HPV infection and the natural history of cervical neoplasia have resulted in the addition of the HPV DNA test along with the Pap test.

From the meta-analysis of the most authoritative published studies [5-8] it can be concluded that the sensitivity of Pap test combined with the HPV DNA test is higher than the sensitivity of each individual method. This observation suggests that the two methods complement each other effectively. In contrast, the specificity of the Pap test combined with the HPV DNA test was lower than the ratings of the two methods separately as they differ in sensitivity and specificity $[9,10]$. Regarding the positive predictive value (PPV) the findings are equivocal: some studies report that the values of PPV were similar for each method separately and for their combination, while others report smaller values of PPV for their combination. As expected, the negative predictive value (NPV) of HPV DNA test in conjunction with the Pap test was high and some studies report values of almost $100 \%$.

In the recent years, new technologies for cervical cancer detection have been promoted to physicians and the public. Some studies proposed the shift from DNA detection to mRNA identification of the viral E6/E7 oncogenes that are linked to oncogenic activation. Among them, mRNA typing with nucleic acid sequence based amplification (NASBA) [1113] and flow cytometry (mRNA-Flow-FISH) techniques for E6/E7 HPV mRNA detection have been enrolled in cancer and precancerous lesions' detection with promising results in increasing PPV and reducing unnecessary recalls and referrals to colposcopy [14-18]. At the same time, it seems that the immunocytochemical detection of genetic effects such as overexpression of p16 is a methodology which can increase the diagnostic accuracy of the Pap test $[19,20]$.

Several published studies in the literature are attempting to clarify the role of each technique as a unique test to substitute or replace the Pap test $[5-8,11,14-24]$. By the detailed analysis of the published studies it can be concluded that the performance of the methods under control differ significantly, affected by the disease incidence and the prevalence of HPV infection in the population study group, resulting in that the individual application of one method, even if it offers a level of protection, does not reliably determine the risk of each individual woman.

Advances in the areas of computer science and artificial intelligence allow the development of computer systems that support clinical diagnosis or therapeutic and treatment decisions based on individualised patient data $[25,26]$. Clinical decision support systems (CDSSs) aim to codify and strategically manage biomedical knowledge to handle challenges in clinical practice using mathematical modelling tools, medical data processing techniques, and artificial intelligence methods. CDSSs cover a wide range of applications, from diagnosis' support to modelling the probability of occurrence of various diseases or the efficiency of alternative therapeutic schemes. To do so, they are using not only individual patient data but also data on risk factors and efficiency of available therapeutic schemes stored in databases. CDSSs are based on statistical analysis methods, such as regression analysis, or artificial intelligence techniques, such as artificial neural networks (ANNs) and pattern recognition techniques [27]. These can be used in order to extract hidden information with essential clinical value from large datasets. Based on complex algorithms, CDSSs may combine in a nonlinear complex way a number of characteristics, for example, data related to the patient (epidemiologic data, medical history, etc.), data related to the disease (examinations' results, biomarkers, course of the disease, etc.), or data related to the treatment (drug selection, drug doses, etc.). In this way, CDSSs provide clinicians with patient-specific assessments or recommendations to aid clinical decision making, or, even more, to provide predictions of diagnostic or prognostic outcomes.

Regarding cervical cancer, an intelligent decision making system may support physicians to improve the selection of protocols for monitoring, diagnosing, and treating women with intraepithelial lesions or cervical cancer or even support the rational selection and the patient-specific follow-up decision making for women who have been treated for highgrade lesions. The majority of published studies, regarding intelligent systems for cervical cancer support, are concerned about computer aided diagnosis systems based on either cytology or colposcopy image analysis [28-31]. On the other hand, various papers have been published in the past few years concerning bioinformatics' CDSSs based on ANNs for cancer improved detection, treatment, and follow-up support [32-37]. To the best of our knowledge, however, a similar bioinformatics intelligent CDSS for supporting and improving cervical cancer detection and triage, like the proposed system, has not been reported in the literature.

This study aims to investigate the potential role of a novel intelligent bioinformatics CDSS which intelligently combines the results of various diagnostic techniques used in the modern cytopathology laboratory in order to provide clinicians with patient-specific predictions of diagnostic or prognostic outcomes and thus to identify women at true risk of developing cervical cancer. The preliminary results suggest that the proposed system may improve the accuracy of diagnosis and in comparison to other combinatorial approaches produces the most balanced results in terms of specificity, sensitivity, PPV, and NPV.

\section{Materials and Methods}

2.1. Clinical Data. Data have been collected randomly from women enrolled in a research project conducted by the Department of Cytopathology of the Medical School of Athens University ("ATTIKON" University Hospital) and the Department of Obstetrics and Gynaecology of the University Hospital of Ioannina. Our study has been approved by the Bioethics Committee of the "ATTIKON" University Hospital and the Bioethics Committee of the University Hospital of Ioannina. Participating women had signed an informed patient consent (ICON) form allowing the use of their epidemiologic, diagnostic, and molecular data for the needs of the system's development and research. The clinical data (molecular examinations' results, cytological diagnoses, 
histological examination of biopsies, visit number and date, patient age, etc.) have been registered and stored into a database which has been developed for the research project purposes. For the processing of the data and algorithm training and testing, anonymised data were extracted from the database.

The study was carried out on ThinPrep LBC specimens obtained from women referred for colposcopy for two reasons: (1) either because they had a previous abnormal Pap test, or (2) they volunteered to participate in the study and accepted a colposcopical examination as well as the application of various biomarkers on their biological material even if they had a normal Pap test (e.g., women with HPV). In case that a negative Pap test was followed by negative colposcopy, no biopsy was taken and the case was considered as clinically negative. The smears were routinely prepared for cytological examination and the remaining material was used for evaluation of additional biomarkers (reflex tests). The cytology was assessed by experienced cytopathologists. Biopsies were obtained from samples during colposcopy and/or from surgical specimens through conization and were fixed and prepared according to standard histopathology protocols. All cases except the clinically negative ones were diagnosed by a single expert histopathologist as daily routine diagnosis and the evaluation of the biopsy was blinded from the results of cytology and other ancillary tests. The histopathologist uses as standard procedure p16 test (CINtec) in all histological material from the cervix.

The patients' database includes more than 5,000 patients with more than 10,000 tests' series due to follow-up cases. Each of these series includes the following tests: cytology according to the revised Bethesda classification (TBS2001 system) [38, 39]; HPV DNA typing using the CLART HUMAN PAPILLOMAVIRUS 2 (GENOMICA) that allows simultaneous detection of 35 different HPV genotypes (both high and low risk) by PCR amplification of a fragment within the highly conserved L1 region of the virus [40]; NASBA assays [41] (NucliSENS EasyQ HPV v1.0) that are used for the identification of E6/E7 mRNA of the HPV types 16, 18, 31, 33, and 45; the PermiFlow (Invirion Diagnostics, LLC, Oak Brook, IL) that allows the identification of E6/E7 mRNA expression of high-risk HPV using flow cytometry technique [17]; and finally the immunocytochemical expression of p16 using the CINtec Cytology Kit [42]. All these examinations produce results that can be used in a classification process and they provide assessments of the clinical cytological sample as a whole and not of individual cells.

Cytological findings of each patient were interpreted according to the Bethesda classification system and were classified as follows: (a) within normal limits (WNL); (b) atypical squamous cells of undetermined significance (ASCUS); (c) low-grade squamous intraepithelial lesion (LSIL); (d) highgrade squamous intraepithelial lesion (HSIL); (e) squamous cell carcinoma (SCC) or adenocarcinoma (AdenoCa). Regarding the HPV DNA test, for which the cytology laboratory is accredited by WHO and is proficient for the specific technique, we considered high-risk (HR) HPV types as HPV types $16,18,26,31,33,35,39,45,51,52,53,56,58$, $59,66,68,73,82$, and 85; and low-risk (LR) HPV types as
HPV types 6, 11, 40, 42, 43, 44, 54, 61, 62, 70, 71, 72, 81, 83, 84, and $89[3,4]$. It is well known that the probability of a lowrisk subtype to cause cervical lesions is very small; however, the specific HPV DNA test is simultaneously identifying both high-risk and low-risk HPV subtypes and thus we used all available typing results during the development of the system, in order to evaluate its performance based on all available information.

For the cases that had histological outcome, the histological diagnosis was used as the golden standard. Random biopsies were not obtained in clinically negative cases, which are defined as cases that had negative cytology and additionally negative colposcopy; for these cases it is not allowed by the ethical committee to have a sample for histological examination. These cases may introduce a small bias in the interpretation of the cytology performance. A cervical biopsy was performed if Pap test revealed ASCUS and above cytological categories (ASCUS+) or there was a visible lesion upon colposcopy. Biopsy was performed by experienced colposcopists (in practice for more than 10 years) as part of the study protocol. The three-tiered cervical intraepithelial neoplasia (CIN) grading system was used for histological diagnosis; thus the cases with histology were classified as follows: (a) without evidence of malignancy (negative histology); (b) cervical intraepithelial neoplasia grade I (CIN1); (c) cervical intraepithelial neoplasia grade II or III (CIN2/3); (d) squamous cell carcinoma (SCC) or adenocarcinoma (Adeno-Ca).

From the more than 10,000 tests' series, 740 cases that were fully completed were selected for examination in this study and were further analysed (Table 1). Cases with one or more missing or invalid/inadequate tests' results were excluded from the study.

For each of the 740 cases, a feature set consisting of 46 variables derived by the applied tests was created. The result of the cytological examination has been used according to the Bethesda system. Results of the HPV DNA test examination were expressed as 35 individual variables (either positive or negative), one for each HPV DNA genotype. For the EasyQ test (NASBA) we used the result for each individual HPV type $(16,18,31,33$, and 45$)$. The result of the PermiFlow test (FLOW) was used as positive or negative, as was the result of the immunocytochemical expression of p16. Additionally, other variables expressing the HPV DNA test results were added; for instance, the existence of high-risk or low-risk types was expressed as either positive or negative. Table 2 shows in detail the 46 independent variables/features that were collected for each case.

According to the final outcome (the histological examination or a clinically negative result), each of the 740 cases was classified into the following classes: class 1: negative or clinically negative, class 2: CIN1, class 3: CIN2 or CIN3 (CIN2/3), and class 4: cancer (SCC or Adeno-Ca).

2.2. Feature Selection. The first step of our study was to employ feature selection algorithms in order to identify which of the 46 aforementioned features contributes most to the prediction of the underlying condition of each woman, that is, 
TABLE 1: Correlation between the cytological and histological findings of the dataset used in the study.

\begin{tabular}{|c|c|c|c|c|c|c|}
\hline & \multicolumn{5}{|c|}{ Pap test result } & \multirow{2}{*}{ Total } \\
\hline & WNL & ASCUS & LSIL & HSIL & SCC/Adeno-Ca & \\
\hline \multicolumn{7}{|c|}{ Histological examination result } \\
\hline Clinically negative & 196 & 0 & 0 & 0 & 0 & $196(26.5 \%)$ \\
\hline Negative & 35 & 60 & 22 & 5 & 0 & $122(16.5 \%)$ \\
\hline CIN1 & 31 & 66 & 142 & 22 & 0 & $261(35.3 \%)$ \\
\hline $\mathrm{CIN} 2 / 3$ & 3 & 13 & 27 & 93 & 0 & $136(18.4 \%)$ \\
\hline SCC/Adeno-Ca & 0 & 1 & 2 & 7 & 15 & $25(3.4 \%)$ \\
\hline Total & $265(35.8 \%)$ & $140(18.9 \%)$ & $193(26.1 \%)$ & $127(17.2 \%)$ & $15(2 \%)$ & 740 \\
\hline
\end{tabular}

WNL: within normal limits, ASCUS: atypical squamous cells of unknown significance, LSIL: low-grade squamous intraepithelial lesion, HSIL: high-grade squamous intraepithelial lesion, SCC: squamous cell carcinoma, Adeno-Ca: adenocarcinoma, CIN: cervical intraepithelial neoplasia.

the class of each case. The feature selection (FS) [43] problem in pattern recognition may be stated as follows: given a set of $N$ features, find the best subset consisting of $l$ features that contribute most to classification/prediction accuracy.

Feature subset selection algorithms can be classified into two main categories: the filter approach and the wrapper approach. In the filter approach the FS is done independently of the learning algorithm of a classifier. A class separability measuring criterion $C(k)$ is adopted in order to rank all the features. The value of the criterion $C(k)$ is computed for each of the features $k=1,2, \ldots m$. Features are then ranked in the order of descending values of $C(k)$. The $l$ features corresponding to the $l$ best values of the specific criterion are selected to form the best subset. In wrapper type methods, the FS is "wrapped" around a learning method: the usefulness of a feature subset is directly judged by the estimated accuracy of a trained classifier. For high-dimensional datasets, wrapper methods are far too computationally expensive to be used because each feature subset considered must be evaluated with the trained classifiers. For this reason, wrapper methods will not be considered in this study.

In order to perform the FS task, we combined two different filter methods [44]. The area between the empirical receiver operating characteristic (ROC) curve and the random classifier slope has been proposed as a class separability measuring criterion [43]. As presented in [43], it measures the overlap between the probability density functions describing the data distribution of a feature in two classes. This criterion serves as a measure of the class discrimination capability of a specific feature. The second filter method we applied is the recently proposed minimum redundancy-maximum relevance (MRMR) feature selection framework, a mutual information based methodology, which has proved to be one of the best filter methods [45]. Both methods return the best feature subset for a selected value $l$.

During feature ranking using the ROC FS method, we applied an ad hoc cross correlation technique that incorporates correlation information into the ranking procedure so as to avoid the existence of correlated features into the best subset. The cross correlation coefficient between features is considered in order to exclude features which are correlated with the top-ranked. This technique is described in detail in
[43]. Using this procedure we are able to define the maximum number of the $l$ best features.

Both of the adopted FS methods measure the classification capability of a specific feature with respect to a twoclass problem. Since our dataset consists of 4 classes, in order to perform the FS task, we adopted a similar to the "one against one" multi-class classification strategy. Thus, we split the dataset into 6 splits, one for each pair of classes; then we applied the 2 mentioned FS techniques to each split and lastly we explored the common top-ranked features between the 12 best feature subsets returned by the two FS techniques. Ultimately, these $l$ common top-ranked features form the best feature subset which was used for the development of the presented system.

\subsection{Classification/Prediction Models and System's Architecture.} The development of an intelligent clinical decision support system involves the employment of several machine learning methods and pattern recognition/classification techniques. Machine learning is concerned about the development and the study of intelligent systems that can learn from data, while pattern recognition/classification aims to use these systems in order to classify an object into a correct class based on features characterising the object. These features are the ones provided by the FS task. The machine learning algorithms that implement pattern classification are known as classifiers. There is a variety of classifiers used for pattern classification; each one of those has certain limitations and advantages. There are simple classifiers like the $k$-nearest neighbours $(k$ $\mathrm{NN}$ ) and the Bayesian classifier and more complex ones like the artificial neural networks (ANNs) [27, 43, 46, 47]. For example, the $k$-NN may be considered as a simple classifier because it classifies a sample based on the $k$-closest training samples in the feature space; it just computes the distances between the new sample and the samples of the training set and according to these distances it classifies the sample to the class of the closest training samples. On the contrary, ANNs are complex networks of artificial neurons interconnected with each other, which obtain knowledge and the ability to classify a sample by the application of complex learning algorithms. This capability of learning from a certain dataset makes the neural networks suitable for classification and prediction tasks in practical situations. Furthermore, neural 
TABLE 2: Variables characterising patients' biological status.

\begin{tabular}{|c|c|c|}
\hline Variable name & Description & Value range \\
\hline Pap test & $\begin{array}{l}\text { The result of the cytological } \\
\text { examination expressed according to } \\
\text { Bethesda system }\end{array}$ & $\begin{array}{l}1: \text { WNL, } 2: \text { ASCUS, } 3: \text { LSIL, } \\
4: \text { HSIL, } 5: \text { SCC or ADENO-Ca }\end{array}$ \\
\hline $\begin{array}{l}\text { HPV DNA Arrays: HPV-6, HPV-11, HPV-16, } \\
\text { HPV-18, HPV-26, HPV-31, HPV-33, HPV-35, } \\
\text { HPV-39, HPV-40, HPV-42, HPV-43, HPV-44, } \\
\text { HPV-45, HPV-51, HPV-52, HPV-53, HPV-54, } \\
\text { HPV-56, HPV-58, HPV-59, HPV-61, HPV-62, } \\
\text { HPV-66, HPV-68, HPV-70, HPV-71, HPV-72, } \\
\text { HPV-73, HPV-81, HPV-82, HPV-83, HPV-84, } \\
\text { HPV-85, HPV-89 }\end{array}$ & $\begin{array}{l}\text { The existence of individual subtypes } \\
\text { according to the HPV DNA } \\
\text { examination }\end{array}$ & $\begin{array}{l}0 \text { if the specific subtype is not } \\
\text { found, } 1 \text { if the specific subtype is } \\
\text { found }\end{array}$ \\
\hline HR-HPV DNA & $\begin{array}{l}\text { The existence of high-risk subtypes } \\
\text { found by the HPV DNA test }\end{array}$ & $\begin{array}{l}0 \text { if none of the high-risk types } \\
\text { was found, } 1 \text { if at least one of the } \\
\text { high-risk types is found }\end{array}$ \\
\hline LR-HPV DNA & $\begin{array}{l}\text { The existence of low-risk subtypes } \\
\text { found by the HPV DNA test }\end{array}$ & $\begin{array}{l}0 \text { if none of the low-risk types is } \\
\text { found, } 1 \text { if at least one of the } \\
\text { low-risk types is found }\end{array}$ \\
\hline Arrays number & $\begin{array}{l}\text { The number of HPV subtypes found } \\
\text { by the HPV DNA test }\end{array}$ & Expressed as number \\
\hline N16 & $\begin{array}{l}\text { The result of the NASBA mRNA test } \\
\text { for HPV subtype } 16\end{array}$ & 0 if negative, 1 if positive \\
\hline N18 & $\begin{array}{l}\text { The result of the NASBA mRNA test } \\
\text { for HPV subtype } 18\end{array}$ & 0 if negative, 1 if positive \\
\hline N31 & $\begin{array}{l}\text { The result of the NASBA mRNA test } \\
\text { for HPV subtype } 31\end{array}$ & 0 if negative, 1 if positive \\
\hline N33 & $\begin{array}{l}\text { The result of the NASBA mRNA test } \\
\text { for HPV subtype } 33\end{array}$ & 0 if negative, 1 if positive \\
\hline N45 & $\begin{array}{l}\text { The result of the NASBA mRNA test } \\
\text { for HPV subtype } 45\end{array}$ & 0 if negative, 1 if positive \\
\hline FLOW & $\begin{array}{l}\text { The result of the identification of } \\
\text { E6/E7 mRNA expression of high-risk } \\
\text { HPV using flow cytometry technique }\end{array}$ & $\begin{array}{l}0 \text { if negative (positive expression } \\
<1.5 \% \text { ), } 1 \text { if positive (positive } \\
\text { expression }>1.5 \% \text { ) }\end{array}$ \\
\hline p16 & $\begin{array}{l}\text { The result of the p16 } \\
\text { immunocytochemical examination }\end{array}$ & 0 if negative, 1 if positive \\
\hline
\end{tabular}

WNL: within normal limits, ASCUS: atypical squamous cells of unknown significance, LSIL: low-grade intraepithelial lesion, HSIL: high-grade squamous intraepithelial lesion, SCC: squamous cell carcinoma, ADENO-Ca: adenocarcinoma.

networks are inherently nonlinear which makes them more suitable for processing complex data patterns, in contrast to many traditional methods.

In this study, in order to develop the proposed CDSS, we employed and tested 6 classifiers: the $k$-nearest neighbours $(k-\mathrm{NN})$ classifier [46], the naïve Bayesian (NB) classifier [43], the classification and regression tree (CART) [48], and 3 types of ANNs, namely, the multilayer perceptron network (MLP) [47], the radial basis function network (RBF) [47], and the probabilistic neural network (PNN) $[49,50]$.

The classifiers were designed to classify the cases into the following 4 groups corresponding to the cervical histology: negative, CIN1, CIN2/3, and cancer (SCC or Adeno-Ca). The feature subset characterising each case, which is derived by the FS task, was used as the input of each classifier. Thus, each classifier takes as inputs results from the examinations of each case and outputs its classification group, providing in this way a prediction regarding the actual cervical status of each woman. The available dataset (Table 1) was divided into 3 sets: the training set (486 cases) which was used to build and train the classifiers, the validation set (126 cases) which was used to optimise the parameters of each classifier, and the test set (128 cases) which was used to evaluate their predictive performance. The 3 sets were properly stratified so that the classes' distribution in each set is approximately the same as that in the initial dataset. Thus, each set contains representative samples of the same larger population. We have also to note that, due to the many different classes and the diversity of the examinations' results, we had to use a large set for training ( $66 \%$ of the initial dataset) so as to provide to the classifiers representative samples of every situation. During the training phase, the cases of the training set are provided to the classifiers along with their classes (their actual histology) and the classifiers learn from this specific dataset using a learning algorithm. The cases of the validation and the test sets are not used during training; thus these cases are 
unknown to the classifiers (unseen data). The validation set is used as a test platform for fine tuning model's parameters and selecting the best-performing model, while the test set is used to assess the predictive performance of the developed models on data which have not been used in any way in the designing process.

Through a process of designing, training, and testing the aforementioned classifiers, we tried to investigate the potential role of these models to improve the accuracy of diagnosis. However, no single classifier produced satisfactory results. By thoroughly investigating the classification results for each case separately, we discovered that the PNN demonstrated good predictive performance for most cases with cytology LSIL and above, and specifically for women with cytology LSIL harbouring CIN grade 2 or worse (CIN2+). However, the PNN showed poor performance on identifying the correct histology of women with ASCUS cytology. On the other hand, we discovered that the MLP, even though underperformed regarding the whole dataset, produced good prediction outcomes for the cases with ASCUS cytology. This fact led us to design a hybrid architecture, by combining a PNN and a MLP.

The presented CDSS consists of two subsystems: the main subsystem is a PNN, while the secondary is a MLP. The PNN is used for the management of all the cases excluding those with Pap test ASCUS, while the MLP is used for the management of the ASCUS cases only. According to the value of the Pap test, each case is promoted to the main or the secondary subsystem; if Pap test is ASCUS, the data are promoted to the MLP; otherwise they are promoted to the PNN. The schematic diagram of the proposed decision support system is presented in Figure 1. This architecture proved to provide better classification results comparing to a single classifier approach.

The learning and the predictive ability of an ANN is determined by several factors, such as the type of the network and the parameters of the specific type, the network's architecture (topology), the learning algorithm chosen for training, and the characteristics of the data provided to the network. Therefore, in order to construct the CDSS, the task of identifying the optimal architecture and parameters of the PNN and the MLP has to be accomplished.

2.3.1. The Probabilistic Neural Network (PNN). A probabilistic neural network is a kind of multilayer feed-forward radial basis artificial neural network suitable for classification and prediction problems. PNNs are widely used for pattern recognition problems, nonlinear mapping, and estimation of the probability of class membership and likelihood ratios. The PNN is based on the theory of Bayesian classification and is closely related to classical estimators for probability density functions $[49,50]$. The basic operation performed by the PNN is an estimation of the probability density function of each class from the provided training samples using Gaussian kernels. These estimated densities are then used in a decision rule to perform the classification.

The PNN architecture consists of four layers: the input layer, the pattern layer, the summation layer, and the output layer, as depicted in Figure 2. The number of neurons of the two hidden layers (pattern and summation layers) is determined by the training set. The pattern layer contains one neuron for each sample of the training set, while the summation layer contains one neuron for each class of the training set. The training process of the PNN is straightforward and it is accomplished by setting the weights of the network using the samples of the training set; thus no learning algorithm is applied during PNN's implementation. The weights between the input and the pattern layer are set as follows:

$$
w_{i j}^{(P)}=p_{i j}
$$

where $w_{i j}^{(P)}$ is the weight between the $i$ th neuron of the input layer and $j$ th neuron of the pattern layer, and $p_{i j}$ is the value of the $i$ th feature of the $j$ th sample of the training set. The weights between the pattern and the summation layer are set as follows:

$$
w_{j k}^{(S)}= \begin{cases}1 & \text { if } T_{k}^{(j)}=1 \\ 0 & \text { else }\end{cases}
$$

where $w_{j k}^{(S)}$ is the weight between the $j$ th neuron of the pattern layer and $k$ th neuron of the summation layer. The value of $T_{k}^{(j)}$ is 1 only when sample $j$ is associated with class $k$ and 0 elsewhere.

When an input is presented, the pattern layer computes the distances between the input vector and the training vectors and produces a vector which indicates how close the input is to the training samples, as follows:

$$
d_{j}^{(P)}=\sqrt{\sum_{i}\left(w_{i j}^{(P)}-x_{i}\right)^{2}},
$$

where $d_{j}^{(P)}$ is the distance between the input vector and the $j$ th sample of the training set, while $x_{i}$ denotes the $i$ th variable of the input ( $i$ th node of input layer).

The transfer function of the neurons of the pattern layer is a radial basis function. The output of each pattern neuron is computed as

$$
P_{j}=\exp \left(-\frac{d_{j}^{(P)}}{2 \sigma^{2}}\right),
$$

where $\sigma$ is a smoothing parameter corresponding to the standard deviation of the Gaussian distribution.

Each summation neuron sums the contributions for each class of the input to produce at the net output a vector of probabilities. The output of each summation node can be expressed as

$$
S_{k}=\frac{1}{\sum_{j} w_{j k}^{(S)}} \sum_{j} w_{j k}^{(S)} \cdot P_{j} .
$$

Finally, a competitive transfer function in the output layer (single neuron) classifies the input vector into a specific 


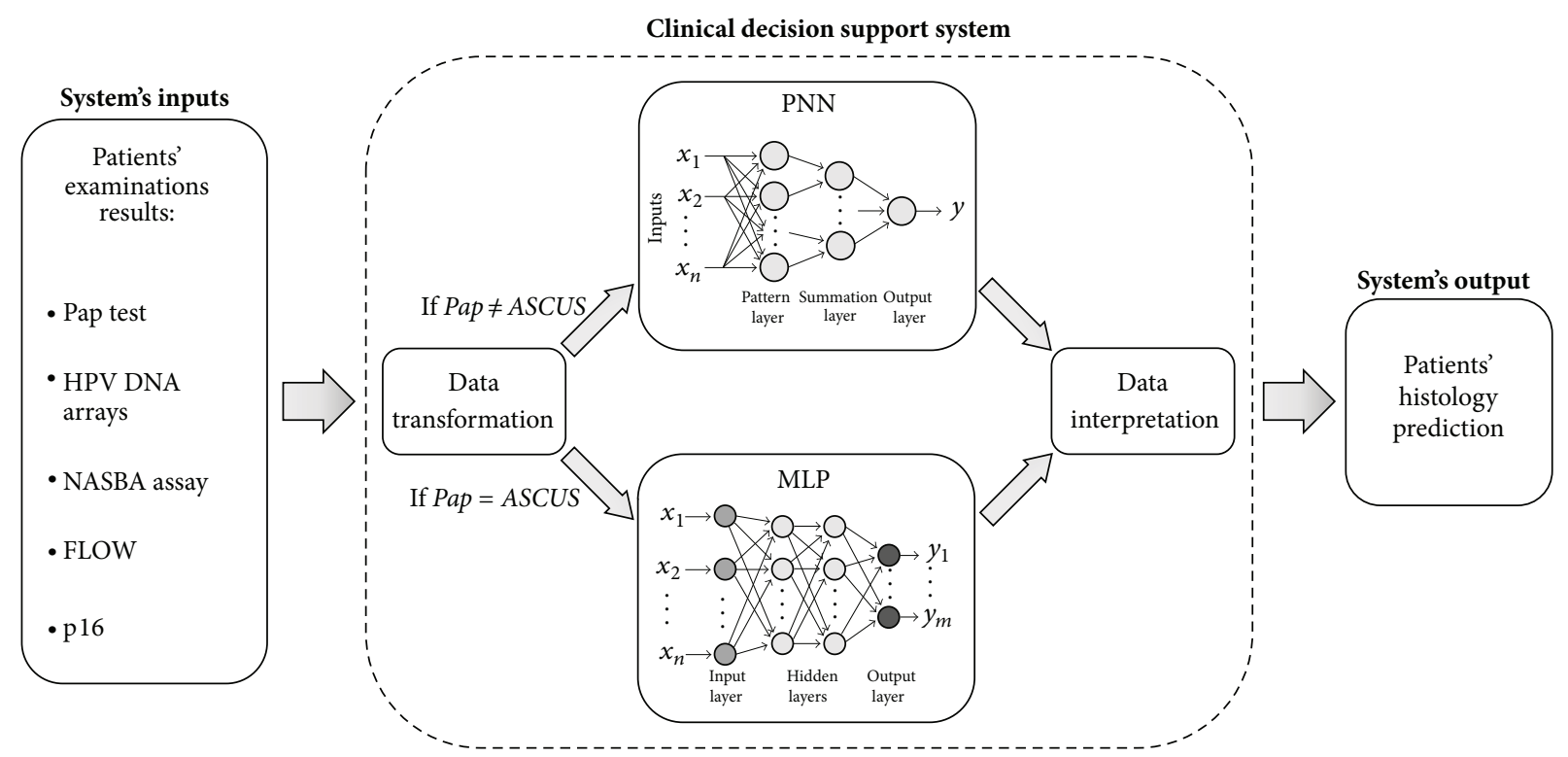

FIGURE 1: Schematic block diagram of the proposed decision support system. The examinations' results of each patient are used as inputs to the CDSS. The medical information is transformed to data appropriate for processing by the PNN and the MLP subsystems. According to the value of the Pap test, the transformed data of each case is promoted to the PNN or the MLP subsystem; if Pap test is ASCUS, the data are promoted to the MLP; otherwise they are promoted to the PNN. The output of each network is properly transformed by the data interpretation block to medical information. At the end, the CDSS provides predictions regarding the actual cervical status of each woman. NASBA: nucleic acid sequence based amplification for the identification of E6/E7 mRNA of the HPV types 16, 18, 31, 33, and 45; FLOW: flow cytometric E6/E7 HPV mRNA assay; p16: p16 immunocytochemical examination; ASCUS: atypical squamous cells of unknown significance; PNN: probabilistic neural network; MLP: multilayer perceptron network.

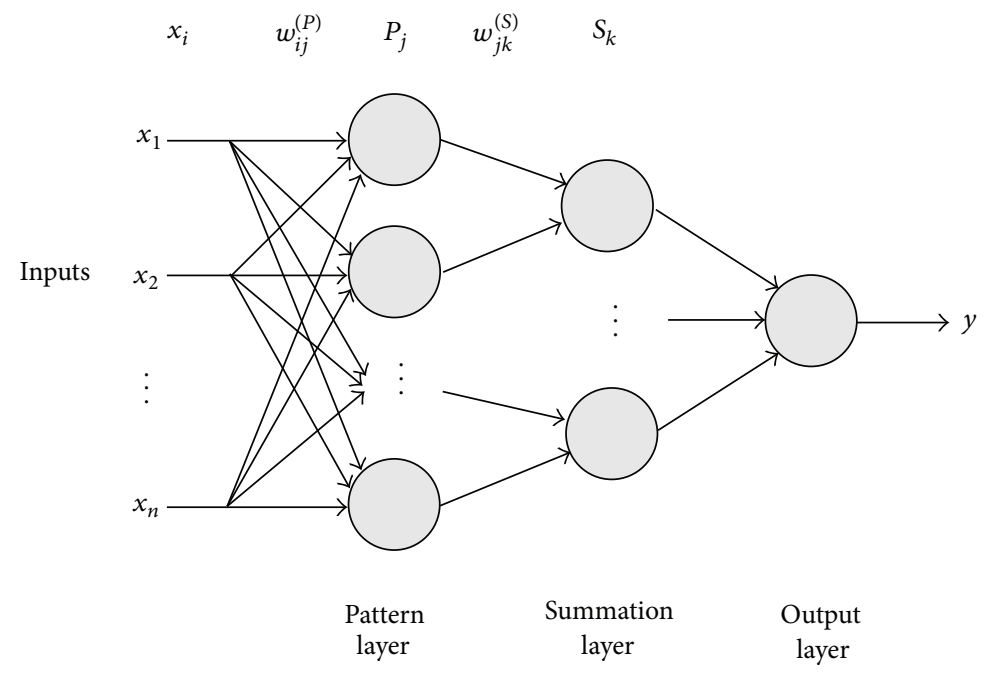

FIgURE 2: Architecture of a probabilistic neural network (PNN).

class if that class had the maximum output value from the corresponding neuron at the summation layer:

$$
y=\arg \max _{k} S_{k} .
$$

From the above, it is obvious that in the PNN architecture the number of the hidden layers and the transfer functions of the neurons are predefined and the number of the neurons of each hidden layer depends on the size and the form of the training set. The single free parameter of this network is the smoothing parameter sigma $(\sigma)$, the standard deviation of the Gaussians. Thus, the selection of the optimal PNN, which will constitute the main subsystem of the CDSS, is essentially the act of determining the optimal sigma value.

2.3.2. The Multilayer Perceptron Network (MLP). The multilayer perceptron is the most widely used neural network. It is a feed-forward ANN with an input layer that receives external inputs, one or more hidden layers and an output 
layer providing the output of the network. Each layer of the MLP includes one or more neurons directionally linked with the neurons from the previous and the next layer. Determining the right architecture of a MLP is the task of selecting the optimal parameters of the network, such as the number of the hidden layers and the number of the neurons of each hidden layer. As far as the learning algorithm is concerned, the back-propagation (BP) algorithm [47] is the most common learning technique for training a typical MLP. During training with the $\mathrm{BP}$ algorithm, information about the errors of the network on known data is propagated backwards from the output layer to the input layer and it is used to adjust the connections between the layers and their neurons (the weights and biases of the network) in order to minimize the error and thus improve performance.

As far as the training of the MLPs is concerned, the Levenberg-Marquardt BP algorithm was used for the learning process, while the mean squared normalized error (MSE) was used as the network's cost function [47]. Training a MLP is essentially the process of modifying the weights and biases of the network in order to minimize this cost function. The learning rate and the momentum of the BP algorithm were set equal to 0.1 and 0.9 , respectively. In order to avoid overfitting, an early-stopping learning technique was implemented, according to which the classification error on the validation subset was monitored during the training process. When the validation error increases for a specified number of iterations of the $\mathrm{BP}$ algorithm, the training is stopped, and the weights and biases at the minimum of the validation error are returned. For the training process, criteria for convergence were met with 40 maximum validation failures or when MSE $\leq 0.0001$ or when a maximum of 1000 iterations (epochs) was reached.

As discussed in [51], empirical studies often found that networks with many hidden layers generally perform no better, and often worse, than neural networks with one or two hidden layers. Thus, in this study, we considered MLP architectures with one or two hidden layers. All neurons in the hidden and the output layers use the sigmoidal activation function. In order to identify the optimal network topology, we applied a trial-and-error cascade constructive process by adding neurons to the hidden layers, one at time, and evaluating the developed MLPs. For each MLP architecture (one-hidden-layered and two-hidden-layered), this process was stopped when the MLPs showed continuous impaired performance.

With the determined number of hidden layers and neurons, both the learning rate and the momentum coefficient of the $\mathrm{BP}$ algorithm were further investigated to ensure a high probability of global network convergence.

2.3.3. Selection of the Optimal Models. In order to identify the optimal parameters of the developed classifiers and eventually select the optimal PNN and MLP which comprise the CDSS's subsystems, we performed the parameter tuning task by evaluating the developed models on the validation set. However, during this task we discovered that classifiers with different parameters presented the same best performance on the validation set, making it difficult to select the optimal models. Thus, in order to perform more accurately the model selection task, we also took into consideration the classification performance on the training set.

Let the classification error on the training set (resubstitution error) be denoted as $\varepsilon^{r}$ and the classification error on the validation set as $\varepsilon^{v}$. For each classification algorithm used in this study, we build $S$ classifiers with different parameters, and we define as optimal the classifier which minimizes the cost function:

$$
J_{m}=\frac{\varepsilon_{m}^{r}+\varepsilon_{m}^{v}}{2}, \quad \forall m \in S, \forall \varepsilon_{m}^{v} \equiv \min \left\{\varepsilon_{m}^{v}\right\}
$$

Utilizing the above cost function for the selection of the optimal parameters, we ensure that the optimal models demonstrate the best predictive performance on the validation set (the models which minimize the cost function must produce the minimum classification error on the validation set) and at the same time they perform well on the cases of the training set (the models which minimize the cost function must produce low resubstitution error).

2.3.4. Performance Evaluation. The final performance evaluation task was carried out using resubstitution and holdout validations. In resubstitution validation, the model is tested on the data which have been used in the learning process, that is, the data of the training set. This method provides a measure of the network's learning ability; yet it is not preferable for performance evaluation tasks as it is known to be optimistically biased. However, as shown in $[52,53]$, in discrete classification problems with large-sample categorical datasets, like the classification problem of this study, resubstitution can be significantly accurate relative to more complex error estimation schemes, since the optimistic bias and the variance of the method tend to be vanished as the sample size increases, provided that classifier complexity is not too high. For this reason we decided to take into consideration the performance of the final models when for testing the training and the validation sets are used. In holdout validation the optimal models are tested on data that were not used in any way in the building process (training and parameter tuning), that is, the data of the test set. The holdout classification error serves as a measure of the model's predictive (generalization) ability.

We have to note that we did not use complex error estimation schemes like the $k$-fold cross validation, so as to be able to study the classification result for each case separately and thus to evaluate the system at individual level. It must be noted that for the clinicians the significance of such a system stands on its ability to correctly identify cases with conflicting tests' results which are difficult to be evaluated by them. Hence, more than the overall accuracy, what is important is the correct identification of as many as possible women with insignificant cytological findings harbouring CIN2+ lesions, as well as the correct identification of women with HSIL+ cytology but with actual histology below CIN2.

Due to the above, the predictive performance of the finally selected ANNs is presented by confusion matrices obtained 
TABLE 3: Classification accuracies on the training, validation, and test sets of the 6 classifiers developed (single classifier approach).

\begin{tabular}{|c|c|c|c|c|c|c|}
\hline Classifier & $k-\mathrm{NN}$ & NB & CART & MLP & $\mathrm{RBF}$ & PNN \\
\hline $\begin{array}{l}\text { Optimal } \\
\text { parameters }\end{array}$ & $k=5$ & - & $\begin{array}{c}\text { (i) Pruning level }=7 / 11 \\
\text { (ii) Number of terminal } \\
\text { nodes }=8\end{array}$ & 2 hidden layers $18 \times 18$ & $\begin{array}{l}\text { (i) } 486 \text { neurons in the } \\
\text { hidden layer (all samples } \\
\text { of training set) } \\
\text { (ii) Sigma }=0.6\end{array}$ & Sigma $=0.4$ \\
\hline Training set & $78.6 \%$ & $76.8 \%$ & $77.6 \%$ & $78.4 \%$ & $87.6 \%$ & $87.6 \%$ \\
\hline Validation set & $79.4 \%$ & $80.9 \%$ & $78.6 \%$ & $77.0 \%$ & $77.7 \%$ & $80.2 \%$ \\
\hline Test set & $82.8 \%$ & $82.8 \%$ & $75.8 \%$ & $80.5 \%$ & $70.3 \%$ & $80.0 \%$ \\
\hline
\end{tabular}

$k$-NN: $k$-nearest neighbours classifier, NB: naïve Bayesian classifier, CART: classification and regression tree, MLP: multilayer perceptron network, RBF: radial basis function network, PNN: probabilistic neural network.

through testing the networks on the training, validation, and test sets.

\section{Results}

3.1. Feature Selection. As described, 12 feature subsets have been produced by the application of the adopted FS techniques to the split datasets, 6 of them corresponding to the ROC FS method and 6 to the MRMR FS framework. Incorporating cross correlation information into the ranking procedure of the ROC FS method, we discovered that, from the 46 variables obtained from the 5 medical tests considered, only 24 contain useful uncorrelated information. Investigating the common top-ranked uncorrelated features between the 12 subsets, we concluded that the following 18 contribute importantly to the prediction of the underlying condition of each case: Pap test, HPV-16, HPV-18, HPV31, HPV-33, HPV-45, HPV-51, HPV-53, HPV-58, HR-HPV DNA, LR-HPV DNA, N16, N18, N31, N33, N45, FLOW, and p16. Since the attributes HPV-16, HPV-18, HPV-31, HPV-33, HPV-45, HPV-51, HPV-53, and HPV-58 correspond to highrisk subtypes of the HPV DNA test, we properly transformed the attribute HR-HPV DNA so as to correspond to the existence of the rest high-risk subtypes only. This process took place in order to dismiss the correlation between the HR-HPV DNA attribute and the rest. Eventually, these 18 features form the best feature subset characterizing each patient. Therefore, for each case, the classifiers take as inputs the values of these 18 variables.

\subsection{Classification/Prediction Models Selection and Performance Evaluation}

3.2.1. Single Classifier Approach. As discussed previously, firstly we explored the single classifier approach, according to which a single classifier is being used for the management of all the cases. Six different classifiers have been developed and evaluated. Table 3 presents the classification accuracy on the training, validation, and test sets of each model, along with its optimal parameters. It seems that the single classifier approach is not adequate to address the problem, as none of these classifiers produced satisfactory results. However, by investigating the classification results for each case separately, we discovered that, between these 6 classifiers, the PNN was superior in classifying correctly those cases with Pap test LSIL and above, whereas the MLP was superior in classifying correctly those cases with Pap test ASCUS. With regard to the cases with negative cytology, all the classifiers produced similar results with none of them performing significantly better compared to the others. Because of these findings, we designed the hybrid solution, by combining a PNN for the classification of all the cases excluding those with cytology ASCUS and a MLP for the classification of the cases with ASCUS cytology, which, according to the following results, proved to be better than the single classifier approach.

3.2.2. Selection and Performance of the Optimal PNN. As mentioned before, in the PNN architecture, the only free parameter is the smoothing parameter sigma $(\sigma)$. Thus, in order to identify the optimal network, we trained and evaluated several PNNs with different sigma values.

As the optimal PNN was used for the management of all the cases excluding those with Pap test ASCUS, in order to train and evaluate the developed PNNs, the 140 cases with ASCUS cytology were excluded from the 3 datasets; thus, 400 cases have been used for training, 100 cases for validation and 100 cases for testing these networks. The training, validation, and test sets of the PNNs are presented in Tables 4, 5, and 6, respectively.

The topology of each of the PNNs developed is described as follows: the input layer consists of 18 nodes, one for each of the features of the feature subset derived by the FS task; the pattern layer contains 400 neurons, one for each of the training samples; and the summation layer contains 4 neurons, one for each class of the training set.

Employing several PNNs with different sigma values, we concluded that performance was decreasing significantly for sigma values greater than 0.8 . In order to obtain the optimal value of the parameter $\sigma$, and thus identify the PNN which performs best, we trained and evaluated 800 PNNs, for $\sigma=$ 0.001 to 0.801 , with a step of 0.001 . It must be noted that PNNs are by design very fast networks and thus the time required to train and test 800 PNNs is not an important issue.

Evaluating the 800 developed PNNs, we concluded that the cost function $J_{m}$ is minimized for a sigma value equal to 0.380 . Thus, the optimal PNN comprising the main subsystem of the CDSS is the PNN with $\sigma=0.380$. The predictive performance of this $\mathrm{PNN}$ is presented through 
TABLE 4: Training set of PNNs (histology and cytology).

\begin{tabular}{|c|c|c|c|c|c|}
\hline & \multicolumn{4}{|c|}{ Pap test result } & \multirow{2}{*}{ Total } \\
\hline & WNL & LSIL & HSIL & SCC/Adeno-Ca & \\
\hline \multicolumn{6}{|l|}{ Histological examination result } \\
\hline Negative/clinically negative & 154 & 16 & 3 & 0 & $173(43.3 \%)$ \\
\hline CIN1 & 21 & 97 & 11 & 0 & $129(32.2 \%)$ \\
\hline $\mathrm{CIN} 2 / 3$ & 2 & 18 & 62 & 0 & $82(20.5 \%)$ \\
\hline SCC/Adeno-Ca & 0 & 1 & 6 & 9 & $16(4.0 \%)$ \\
\hline Total & $177(44.2 \%)$ & $132(33.0 \%)$ & $82(20.5 \%)$ & $9(2.3 \%)$ & 400 \\
\hline
\end{tabular}

TABLE 5: Validation set of PNNs (histology and cytology).

\begin{tabular}{|c|c|c|c|c|c|}
\hline & \multicolumn{4}{|c|}{ Pap test result } & \multirow{2}{*}{ Total } \\
\hline & WNL & LSIL & HSIL & SCC/Adeno-Ca & \\
\hline \multicolumn{6}{|l|}{ Histological examination result } \\
\hline Negative/clinically negative & 40 & 2 & 1 & 0 & $43(43.0 \%)$ \\
\hline CIN1 & 5 & 23 & 5 & 0 & $33(33.0 \%)$ \\
\hline $\mathrm{CIN} 2 / 3$ & 1 & 4 & 15 & 0 & $20(20.0 \%)$ \\
\hline SCC/Adeno-Ca & 0 & 1 & 1 & 2 & $4(4.0 \%)$ \\
\hline Total & $46(46.0 \%)$ & $30(30.0 \%)$ & $22(22.0 \%)$ & $2(2.0 \%)$ & 100 \\
\hline
\end{tabular}

TABLE 6: Test set of PNNs (histology and cytology).

\begin{tabular}{lccccc}
\hline & & \multicolumn{2}{c}{ Pap test result } & Total \\
& WNL & LSIL & HSIL & SCC/Adeno-Ca & \\
Histological examination result & & & & & \\
Negative/clinically negative & 37 & 4 & 6 & 0 & 0 \\
CIN1 & 5 & 22 & 16 & $32(33.0 \%)$ \\
CIN2/3 & 0 & 5 & 0 & 4 & $21(21.0 \%)$ \\
SCC/Adeno-Ca & 0 & 0 & $23(23.0 \%)$ & $4(4.0 \%)$ & $4(4.0 \%)$ \\
\hline Total & $42(42.0 \%)$ & $31(31.0 \%)$ & 100 \\
\hline
\end{tabular}

TABLE 7: Confusion matrix obtained through testing the PNN on the cases of the training set (Table 4).

\begin{tabular}{lcccc}
\hline & \multicolumn{4}{c}{ PNN classification result } \\
& Negative & CIN1 & CIN2/3 & Ca \\
\hline Histological examination result & & & & \\
Negative/clinically negative & 163 & 10 & 0 & 0 \\
CIN1 & 13 & 109 & 7 & 0 \\
CIN2/3 & 1 & 5 & 76 & 0 \\
SCC/Adeno-Ca & 0 & 0 & 4 & 12 \\
\hline
\end{tabular}

confusion matrices obtained by testing the network on the training, validation, and test sets (Tables 7, 8, and 9). The overall classification accuracies of the PNN on the training, validation, and test sets are $90.0 \%, 82.0 \%$, and $84.0 \%$, respectively.

Table 10 depicts the comparison between the cytological diagnosis obtained from the Pap test and the PNN, for all the cases excluding ASCUS. Moreover, by comparing Tables 4-6 (cytological diagnosis) with Tables 7-9 (PNN's classifications), respectively, it can be observed that the PNN
TABLE 8: Confusion matrix obtained through testing the PNN on the cases of the validation set (Table 5).

\begin{tabular}{lcccc}
\hline & \multicolumn{4}{c}{ PNN classification result } \\
& Negative & CIN1 & CIN2/3 & Ca \\
\hline Histological examination result & & & & \\
Negative/clinically negative & 40 & 2 & 1 & 0 \\
CIN1 & 5 & 24 & 4 & 0 \\
CIN2/3 & 1 & 3 & 16 & 0 \\
SCC/Adeno-Ca & 0 & 1 & 1 & 2 \\
\hline
\end{tabular}

TABLE 9: Confusion matrix obtained through testing the PNN on the cases of the test set (Table 6).

\begin{tabular}{lcccc}
\hline & \multicolumn{4}{c}{ PNN classification result } \\
& Negative & CIN1 & CIN2/3 & Ca \\
\hline Histological examination result & & & & \\
Negative/clinically negative & 37 & 5 & 0 & 0 \\
CIN1 & 5 & 24 & 4 & 0 \\
CIN2/3 & 0 & 2 & 19 & 0 \\
SCC/Adeno-Ca & 0 & 0 & 0 & 4 \\
\hline
\end{tabular}


TABLE 10: Diagnostic accuracy of cytology and the PNN for all the cases excluding ASCUS.

\begin{tabular}{|c|c|c|c|c|c|c|}
\hline & \multicolumn{3}{|c|}{ Cytological diagnosis } & \multicolumn{3}{|c|}{ PNN diagnosis } \\
\hline & Training set & Validation set & Test set & Training set & Validation set & Test set \\
\hline \multicolumn{7}{|l|}{ Histology } \\
\hline Negative/clinically negative & $89.0 \%$ & $93.0 \%$ & $88.1 \%$ & $94.2 \%$ & $93.0 \%$ & $88.1 \%$ \\
\hline CIN1 & $75.2 \%$ & $69.7 \%$ & $66.7 \%$ & $84.5 \%$ & $72.7 \%$ & $72.7 \%$ \\
\hline CIN2/3 & $75.6 \%$ & $75.0 \%$ & $76.2 \%$ & $92.7 \%$ & $80.0 \%$ & $90.5 \%$ \\
\hline SCC/Adeno-Ca & $56.3 \%$ & $50.0 \%$ & $100.0 \%$ & $75.0 \%$ & $50.0 \%$ & $100.0 \%$ \\
\hline Average accuracy per set & $80.5 \%$ & $80.0 \%$ & $79.0 \%$ & $90.0 \%$ & $82.0 \%$ & $84.0 \%$ \\
\hline Overall accuracy & \multicolumn{3}{|c|}{$80.2 \%(481 / 600$ cases $)$} & \multicolumn{3}{|c|}{$87.7 \%$ (526/600 cases) } \\
\hline
\end{tabular}

TABLE 11: Training, validation, and test sets of the MLPs (ASCUS cases only).

\begin{tabular}{lcccc}
\hline & Training set & Validation set & Test set & \\
\hline Histological examination result & & & & \\
Negative & 36 & 12 & 12 & $60(42.8 \%)$ \\
CIN1 & 40 & 12 & $66(47.2 \%)$ \\
CIN2/3 & 9 & 2 & 2 & $14(9.3 \%)$ \\
SCC/Adeno-Ca & 1 & 0 & $26(18.6 \%)$ & $1(0.7 \%)$ \\
\hline Total & $86(61.4 \%)$ & & $20.0 \%)$ \\
\hline
\end{tabular}

TABLE 12: Confusion matrix obtained through testing the MLP on the training set of the ASCUS cases.

\begin{tabular}{lcccc}
\hline & \multicolumn{4}{c}{ MLP classification result } \\
& Negative & CIN1 & CIN2/3 & Ca \\
\hline Histological examination result & & & & \\
Negative/clinically negative & 34 & 2 & 0 & 0 \\
CIN1 & 14 & 25 & 1 & 0 \\
CIN2/3 & 2 & 1 & 6 & 0 \\
SCC/Adeno-Ca & 0 & 0 & 1 & 0 \\
\hline
\end{tabular}

TABLE 13: Confusion matrix obtained through testing the MLP on the validation set of the ASCUS cases.

\begin{tabular}{lcccc}
\hline & \multicolumn{4}{c}{ MLP classification result } \\
& Negative & CIN1 & CIN2/3 & Ca \\
\hline Histological examination result & & & & \\
Negative/clinically negative & 11 & 1 & 0 & 0 \\
CIN1 & 4 & 8 & 0 & 0 \\
CIN2/3 & 0 & 1 & 1 & 0 \\
SCC/Adeno-Ca & 0 & 0 & 0 & 0 \\
\hline
\end{tabular}

outperformed cytology, as it correctly classified 240 negative cases (Tables 7-9: $163+40+37$ ), 157 CIN1 cases (Tables 7-9: $109+24+24), 111$ CIN2/3 cases (Tables 7-9: 76+16+19), and 18 Ca cases (Tables 7-9: $12+2+4$ ), comparing to the 231 negative cases (Tables 4-6: $154+40+37$ ), 142 CIN1 cases (Tables 4-6: $97+23+22$ ), 93 CIN2/3 cases (Tables $4-6: 62+15+16$ ), and 15 Ca cases (Tables 4-6: 9+2+4) that cytology correctly detected. In total, the PNN predicted correctly the histology of 526 of the 600 cases, whereas cytology diagnosed correctly 481 of the 600 cases of the available dataset (excluding ASCUS). It is noteworthy that the PNN classified correctly 17 of the 27
TABLE 14: Confusion matrix obtained through testing the MLP on the test set of the ASCUS cases.

\begin{tabular}{lcccc}
\hline & \multicolumn{4}{c}{ MLP classification result } \\
& Negative & CIN1 & CIN2/3 & Ca \\
\hline Histological examination result & & & & \\
Negative/clinically negative & 11 & 1 & 0 & 0 \\
CIN1 & 3 & 11 & 0 & 0 \\
CIN2/3 & 0 & 0 & 2 & 0 \\
SCC/Adeno-Ca & 0 & 0 & 0 & 0 \\
\hline
\end{tabular}

LSIL cases harbouring CIN2/3 (Tables 4-6: $18+4+5=27$ LSIL cases with CIN2/3 histology, Tables 7-9: $5+3+2=10$ of these cases classified from the PNN as CIN1 and the rest 17 classified as CIN2/3) and 7 of the 22 HSIL cases with CIN1 histology (Tables 4-6: $11+5+6=22$ HSIL cases with CIN1 histology, Tables 7-9: $7+4+4=15$ of these cases classified from the PNN as CIN2/3 and the rest 7 classified correctly as CIN1).

3.2.3. Selection and Performance of the Optimal MLP. As mentioned before, the MLP was employed exclusively for the classification of the cases with ASCUS cytology. Table 11 shows in detail the distribution of the ASCUS cases used in the training, validation, and test sets of the MLPs.

Adopting the trial-and-error constructive process described previously, we eventually trained and evaluated 514 MLPs: 30 of them with one hidden layer, with their layer's size ranging from 10 to 40 neurons, and 484 with two hidden layers, with hidden layers' sizes ranging from 5 to 27 neurons.

Based on the experimental results, the optimal architecture of the MLP was found to be a network with two hidden layers, with 11 neurons on the first hidden layer and 17 on 
TABLE 15: Definition of positivity of the medical tests involved in this study for performance evaluation purposes.

\begin{tabular}{ll}
\hline Medical tests & Definition of positivity \\
\hline Pap test (cut-off ASCUS+) & ASCUS or worse \\
Pap test (cut-off LSIL+) & LSIL or worse \\
Pap test (cut-off HSIL+) & HSIL or worse \\
HPV DNA test & Existence of any HPV subtype found by the HPV DNA test \\
HR-HPV DNA & Existence of at least one of the high-risk subtypes found by the HPV DNA test \\
NASBA E6/E7 HPV mRNA test & $\begin{array}{l}\text { Positive result of the E6/E7 HPV mRNA test (NASBA) for any of the HPV } \\
\text { subtypes 16, 18, 31, 33, and } 45\end{array}$ \\
Flow cytometric E6/E7 HPV mRNA assay & Positive result of the identification of E6/E7 mRNA expression of high-risk \\
p16 & HPV using flow cytometry technique (positive expression $>1.5 \%)$ \\
\hline
\end{tabular}

Different positivity thresholds have been taken into consideration for Pap test and HPV DNA test. HR-HPV: high-risk human papillomavirus, ASCUS: atypical squamous cells of unknown significance, LSIL: low-grade intraepithelial lesion, and HSIL: high-grade squamous intraepithelial lesion.

TABLE 16: Diagnostic performance of cytology, biomarkers, and the CDSS to identify high-grade cervical intraepithelial neoplasia or cancer (CIN2+).

\begin{tabular}{|c|c|c|c|c|c|}
\hline Histology endpoint CIN2+ & Sensitivity (\%) & Specificity (\%) & PPV (\%) & NPV $(\%)$ & Youden's index \\
\hline Pap test (cut-off ASCUS+) & 98.1 & 45.3 & 33.3 & 98.9 & 0.43 \\
\hline Pap test (cut-off LSIL+) & 89.4 & 67.0 & 43.0 & 96.0 & 0.56 \\
\hline Pap test (cut-off HSIL+) & 71.4 & 95.3 & 81.0 & 92.3 & 0.67 \\
\hline HPV DNA test & 91.9 & 61.5 & 39.9 & 96.5 & 0.53 \\
\hline HR-HPV DNA & 89.4 & 67.4 & 43.2 & 95.8 & 0.57 \\
\hline NASBA E6/E7 HPV mRNA test & 77.0 & 90.2 & 68.5 & 93.4 & 0.67 \\
\hline Flow cytometric E6/E7 HPV mRNA assay & 93.2 & 81.9 & 58.8 & 97.7 & 0.75 \\
\hline p16 & 58.4 & 92.9 & 69.6 & 88.9 & 0.51 \\
\hline CDSS & 89.4 & 97.1 & 89.4 & 97.1 & 0.87 \\
\hline
\end{tabular}

Statistical measures have been calculated using all the cases of the dataset (Table 1). Histology endpoint is CIN2+ for all cases. Definition of positivity of each medical test is presented in Table 15. For the CDSS, positivity was defined as a classification result of CIN2/3 or cancer. CIN2+: cervical intraepithelial neoplasia grade 2 or worse, PPV: positive predictive value, and NPV: negative predictive value.

the second. The input layer of the MLP consists of 17 nodes, one for each of the 18 features of the feature subset excluding Pap test (as all the cases had ASCUS cytology). The output layer contains 4 neurons, one for each class of the dataset. In addition, by adopting a trial-and-error approach, the network appeared to be more efficient with the learning rate at 0.01 and the momentum at 0.8 .

The overall classification accuracies of the MLP on the training, validation, and test sets are $75.6 \%, 76.9 \%$ and, $85.7 \%$, respectively. Tables $12-14$ present the confusion matrices of the MLP obtained through testing the network on the training, the validation, and the test sets of the ASCUS cases, respectively. Using the optimal MLP, we managed to correctly detect the actual histology of 109 of the 140 ASCUS cases (Tables 12-14). It must be noted that due to positive biomarkers, the MLP detected 9 of the 13 ASCUS cases harbouring CIN2/3 (Tables 12-14: $6+1+2$ ).

3.3. Comparison between the CDSS and the Medical Tests to Detect CIN2+Lesions. In order to evaluate the performance of the proposed CDSS compared to the tests involved in this study, we calculated the sensitivity, specificity, positive predictive value (PPV), and negative predictive value (NPV) of the methods on the basis of detecting high-grade cervical intraepithelial neoplasia and cancer (CIN2+). Moreover, we calculated Youden's index (Sensitivity+Specificity-1) of each method, which is a single statistic measure of a test's performance, used for the evaluation of the overall discriminative power of a test and for comparison of this test with others.

The performance measures have been calculated using all the cases of the dataset ( 740 cases). The cutoff of CIN2+ was used in order to have comparable results between the CDSS and the other medical tests. According to this threshold, the cases with histologic diagnosis of CIN1 and below were considered negative and the cases with histologic diagnosis of CIN2 and above were considered positive. The definition of positivity of each medical test is presented in Table 15. As shown is Table 15, different positivity thresholds have been taken into consideration for the Pap test and the HPV DNA test. As far as the CDSS is concerned, the values of the 18 features characterizing each patient are provided to the system and the latter classifies the case into one of the 4 groups corresponding to cervical histology. For the CDSS, positivity was defined as a classification result of CIN2/3 or cancer. Table 16 presents the diagnostic performance of the CDSS and the medical tests, in terms of sensitivity, specificity, PPV, and NPV, in predicting high-grade cervical intraepithelial neoplasia or cancer. 
TABLE 17: Performance of type "OR" combinations between two tests in detecting CIN2+.

\begin{tabular}{|c|c|c|c|c|c|c|}
\hline Combinations of medical tests & Cytology cutoff & Sensitivity (\%) & Specificity (\%) & PPV (\%) & NPV (\%) & Youden's index \\
\hline Pap test or HPV DNA & ASCUS+ & 99.4 & 37.5 & 30.7 & 99.5 & 0.37 \\
\hline Pap test or HPV DNA & LSIL+ & 96.3 & 52.0 & 35.8 & 98.0 & 0.48 \\
\hline Pap test or HPV DNA & HSIL+ & 96.3 & 60.3 & 40.3 & 98.3 & 0.57 \\
\hline Pap test or HR-HPV DNA & ASCUS+ & 98.8 & 39.2 & 31.1 & 99.1 & 0.38 \\
\hline Pap test or HR-HPV DNA & LSIL+ & 95.7 & 54.7 & 37.0 & 97.8 & 0.50 \\
\hline Pap test or HR-HPV DNA & HSIL+ & 95.7 & 65.8 & 43.8 & 98.2 & 0.62 \\
\hline Pap test or NASBA & ASCUS+ & 98.8 & 44.2 & 33.0 & 99.2 & 0.43 \\
\hline Pap test or NASBA & LSIL+ & 94.4 & 64.6 & 42.6 & 97.7 & 0.59 \\
\hline Pap test or NASBA & HSIL+ & 88.8 & 87.4 & 66.2 & 96.6 & 0.76 \\
\hline Pap test or FLOW & ASCUS+ & 99.4 & 41.8 & 32.2 & 99.6 & 0.41 \\
\hline Pap test or FLOW & LSIL+ & 97.5 & 61.7 & 41.4 & 98.9 & 0.59 \\
\hline Pap test or FLOW & HSIL+ & 96.9 & 80.3 & 57.8 & 98.9 & 0.77 \\
\hline Pap test or p16 & ASCUS+ & 99.4 & 45.3 & 33.5 & 99.6 & 0.45 \\
\hline Pap test or p16 & LSIL+ & 92.5 & 66.1 & 43.2 & 97.0 & 0.59 \\
\hline Pap test or p16 & HSIL+ & 81.4 & 90.2 & 69.7 & 94.6 & 0.72 \\
\hline HPV DNA or NASBA & & 93.8 & 60.3 & 39.6 & 97.2 & 0.54 \\
\hline HPV DNA or FLOW & & 98.1 & 56.6 & 38.6 & 99.1 & 0.55 \\
\hline HPV DNA or p16 & & 93.8 & 59.6 & 39.2 & 97.2 & 0.53 \\
\hline HR-HPV DNA or NASBA & & 91.9 & 65.8 & 42.8 & 96.7 & 0.58 \\
\hline HR-HPV DNA or FLOW & & 97.5 & 61.5 & 41.3 & 98.9 & 0.59 \\
\hline HR-HPV DNA or p16 & & 92.5 & 64.8 & 42.2 & 96.9 & 0.57 \\
\hline NASBA or FLOW & & 96.3 & 79.6 & 56.8 & 98.7 & 0.76 \\
\hline NASBA or p16 & & 87.0 & 85.7 & 62.8 & 95.9 & 0.73 \\
\hline FLOW or p16 & & 96.3 & 77.5 & 54.4 & 98.7 & 0.74 \\
\hline
\end{tabular}

Statistical measures have been calculated using all cases of the dataset (Table 1). Histology endpoint is CIN2+ for all cases. Definition of positivity of each medical test is presented in Table 15. CIN2+: cervical intraepithelial neoplasia grade 2 or worse, HR-HPV: high-risk human papillomavirus, NASBA: nucleic acid sequence based amplification for the identification of E6/E7 mRNA of the HPV types 16, 18, 31, 33, and 45, FLOW: flow cytometric E6/E7 HPV mRNA assay, PPV: positive predictive value, and NPV: negative predictive value.

In addition, we evaluated the performance of the CDSS in comparison to several combinatorial approaches of the medical tests. Two different combinatorial approaches have been taken into consideration; the "logical OR" and the "logical AND" combinations. In "OR" combinatorial approach, the combination is defined as positive when any of the combined tests is positive, while, in "AND" approach, the combination is defined as positive when all of the combined tests are positive. Tables 17, 18, 19, and 20 present the performance of the several combinations considered in detecting CIN2+ lesions.

The CDSS showed high sensitivity (89.4\%), high specificity (97.1\%), high PPV (89.4\%), and high NPV (97.1\%), for detecting CIN2+. In comparison to the medical tests involved in this study and their combinations, CDSS produced the most balanced results in terms of sensitivity, specificity, PPV, and NPV. Moreover, when ranking the tests by maximal Youden's index, which gives equal weight to sensitivity and specificity, the CDSS ranked highest (Youden's index of 0.87 ), outperforming all the tests and their combinations.

\section{Discussion and Conclusions}

The gynaecological smear is viewed as the most successful cancer test of all time and of all organs [54]. Nevertheless, and despite the advances of the last decade, there is still lack of consensus on the optimal management of women with abnormal pap smears; actually a proportion of women having LSIL may have a HSIL and additionally it is not infrequent that women with HSIL cytology may have CIN1 or even normal histology; finally women with ASCUS in cytology present similar problems on their management. There have been many efforts to apply various biomarkers in the triage of abnormal Pap smears [14, 19, 36, 37, 55-65]. The studied methods are either highly sensitive or highly specific, however not both at the same time and thus no perfect method is available today; in our study similar results were found (see Tables 16-20). In our material the percentage of CIN2+ cases in the total of the cases given as ASCUS is (Table $1: 13+1) 14 / 140=10.0 \%$; additionally the percentage 
TABLE 18: Performance of several type "OR” combinations between more than two tests in detecting CIN2+.

\begin{tabular}{|c|c|c|c|c|c|c|}
\hline Combinations of medical tests & Cytology cutoff & Sensitivity (\%) & Specificity (\%) & PPV (\%) & NPV (\%) & Youden's index \\
\hline Pap test or HPV DNA or NASBA & ASCUS+ & 99.4 & 36.6 & 30.4 & 99.5 & 0.36 \\
\hline Pap test or HPV DNA or NASBA & LSIL+ & 96.3 & 51.1 & 35.4 & 98.0 & 0.47 \\
\hline Pap test or HPV DNA or NASBA & HSIL+ & 96.3 & 59.1 & 39.5 & 98.3 & 0.55 \\
\hline Pap test or HPV DNA or FLOW & ASCUS+ & 100.0 & 35.1 & 30.0 & 100.0 & 0.35 \\
\hline Pap test or HPV DNA or FLOW & LSIL+ & 98.1 & 48.9 & 34.8 & 99.0 & 0.47 \\
\hline Pap test or HPV DNA or FLOW & HSIL+ & 98.1 & 56.0 & 38.3 & 99.1 & 0.54 \\
\hline Pap test or HPV DNA or p16 & ASCUS+ & 99.4 & 37.5 & 30.7 & 99.5 & 0.37 \\
\hline Pap test or HPV DNA or p16 & LSIL+ & 96.3 & 51.3 & 35.5 & 98.0 & 0.48 \\
\hline Pap test or HPV DNA or p16 & HSIL+ & 96.3 & 58.9 & 39.4 & 98.3 & 0.55 \\
\hline Pap test or HPV DNA or NASBA or FLOW & ASCUS+ & 100.0 & 34.9 & 29.9 & 100.0 & 0.35 \\
\hline Pap test or HPV DNA or NASBA or FLOW & LSIL+ & 98.1 & 48.7 & 34.7 & 98.9 & 0.47 \\
\hline Pap test or HPV DNA or NASBA or FLOW & HSIL+ & 98.1 & 55.4 & 38.0 & 99.1 & 0.54 \\
\hline Pap test or HPV DNA or NASBA or FLOW or p16 & ASCUS+ & 100.0 & 34.9 & 29.9 & 100.0 & 0.35 \\
\hline Pap test or HPV DNA or NASBA or FLOW or p16 & LSIL+ & 98.1 & 48.0 & 34.4 & 98.9 & 0.46 \\
\hline Pap test or HPV DNA or NASBA or FLOW or p16 & HSIL+ & 98.1 & 54.4 & 37.4 & 99.1 & 0.53 \\
\hline HPV DNA or NASBA or FLOW & & 98.1 & 56.1 & 38.3 & 99.1 & 0.54 \\
\hline HPV DNA or NASBA or p16 & & 93.8 & 58.5 & 38.6 & 97.1 & 0.52 \\
\hline HPV DNA or NASBA or FLOW or p16 & & 98.1 & 54.7 & 37.6 & 99.1 & 0.53 \\
\hline NASBA or FLOW or p16 & & 98.1 & 76.7 & 53.9 & 99.3 & 0.75 \\
\hline
\end{tabular}

Statistical measures have been calculated using all cases of the dataset (Table 1). Histology endpoint is CIN2+ for all cases. Definition of positivity of each medical test is presented in Table 15. CIN2+: cervical intraepithelial neoplasia grade 2 or worse, HR-HPV: high-risk human papillomavirus, NASBA: nucleic acid sequence based amplification for the identification of E6/E7 mRNA of the HPV types 16, 18, 31, 33, and 45, FLOW: flow cytometric E6/E7 HPV mRNA assay, PPV: positive predictive value, and NPV: negative predictive value.

of CIN2+ cases in the total of LSIL cases is (Table 1: $27+2$ ) $29 / 193=15.0 \%$ and both percentages are in agreement with these reported by other researchers in the literature [66]; specifically these are $5-17 \%$ and $9-16 \%$, respectively. On the other hand the percentage of cases given in cytology as HSIL and being lower than CIN2 is (Table 1:22+5) $27 / 127=21.26 \%$, a percentage in agreement with the literature $[67,68]$.

Today, the widely accepted management options of ASCUS and LSIL smears remain either the immediate referral to colposcopy or the cytological surveillance with repeated smears. A policy of immediate referral to colposcopy could potentially result not only in the overloading of colposcopy clinics but also in overtreatment due to subtle colposcopical findings. Many young nulliparous women might be exposed to the physical and psychological sequelae of unnecessary treatment with long-term perinatal morbidity in women being in reproductive age [69-71]. On the other hand, repeating a cervical smear carries the risk of missing highgrade lesions (HSILs), increases nonattending rates (noncompliance [72]), and increases social and psychological burden of women, directly questioning organized screening programs' (OSPs) credibility. Therefore, it is essential to reduce unnecessary colposcopies and, if feasible, to have in advance indication for women treatment, even before the colposcopical examination. Thus, a methodology for more accurate diagnosis is extremely important.
Although HPV related tests may be used in the triage of ASCUS cases $[65,73]$, every effort should be made to develop new tools and biomarkers to improve the accuracy of diagnosis and allow tailored management. Nowadays, there are numerous methods and biomarkers that are available for cervical cancer detection; nevertheless no single method is optimal [56]. Thus, a different approach is required that will be able to combine many parameters in order to produce an accurate risk assessment for each woman. Instead of the futile search for a single golden marker we should evolve current ones and invent more elaborate methods for result evaluation and utilisation. Based on this, we are working since 2010 on an innovative approach of employing advanced mathematical and computing tools for the nonlinear combination of the methods and biomarkers that are available for cervical cancer detection. Up to now preliminary results are presented in the literature $[36,37]$.

The aim of this study was to create a decision support system for the triage of women before referral to colposcopy. This system is based on the standard cytological diagnosis on ThinPrep Pap test smears and the expression of various biomarkers. The preliminary results suggest that the proposed neural network architecture may improve the accuracy of diagnosis; according to Tables 16-20, CDSS provided the 
TABLE 19: Performance of type "AND” combinations between two tests in detecting CIN2+.

\begin{tabular}{|c|c|c|c|c|c|c|}
\hline Combinations of medical tests & Cytology cutoff & Sensitivity (\%) & Specificity (\%) & PPV (\%) & NPV (\%) & Youden's index \\
\hline Pap test and HPV DNA & ASCUS+ & 90.7 & 69.3 & 45.1 & 96.4 & 0.60 \\
\hline Pap test and HPV DNA & LSIL+ & 85.1 & 76.5 & 50.2 & 94.9 & 0.62 \\
\hline Pap test and HPV DNA & HSIL+ & 67.1 & 96.5 & 84.4 & 91.3 & 0.64 \\
\hline Pap test and HR-HPV DNA & ASCUS+ & 88.8 & 73.4 & 48.1 & 95.9 & 0.62 \\
\hline Pap test and HR-HPV DNA & LSIL+ & 83.2 & 79.6 & 53.2 & 94.5 & 0.63 \\
\hline Pap test and HR-HPV DNA & HSIL+ & 65.2 & 96.9 & 85.4 & 90.9 & 0.62 \\
\hline Pap test and NASBA & ASCUS+ & 76.4 & 91.2 & 70.7 & 93.3 & 0.68 \\
\hline Pap test and NASBA & LSIL+ & 72.0 & 92.6 & 73.0 & 92.3 & 0.65 \\
\hline Pap test and NASBA & HSIL+ & 59.6 & 98.1 & 89.7 & 89.7 & 0.58 \\
\hline Pap test and FLOW & ASCUS+ & 91.9 & 85.3 & 63.5 & 97.4 & 0.77 \\
\hline Pap test and FLOW & LSIL+ & 85.1 & 87.2 & 64.9 & 95.5 & 0.72 \\
\hline Pap test and FLOW & HSIL+ & 67.7 & 96.9 & 85.8 & 91.5 & 0.65 \\
\hline Pap test and p16 & ASCUS+ & 57.1 & 92.9 & 69.2 & 88.6 & 0.50 \\
\hline Pap test and p16 & LSIL+ & 55.3 & 93.8 & 71.2 & 88.3 & 0.49 \\
\hline Pap test and p16 & HSIL+ & 48.4 & 98.1 & 87.6 & 87.3 & 0.47 \\
\hline HPV DNA and NASBA & & 75.2 & 91.4 & 70.8 & 93.0 & 0.67 \\
\hline HPV DNA and FLOW & & 87.0 & 86.7 & 64.5 & 96.0 & 0.74 \\
\hline HPV DNA and p16 & & 56.5 & 94.8 & 75.2 & 88.7 & 0.51 \\
\hline HR-HPV DNA and NASBA & & 74.5 & 91.7 & 71.4 & 92.8 & 0.66 \\
\hline HR-HPV DNA and FLOW & & 85.1 & 87.7 & 65.9 & 95.5 & 0.73 \\
\hline HR-HPV DNA and p16 & & 55.3 & 95.5 & 77.4 & 88.5 & 0.51 \\
\hline NASBA and FLOW & & 73.9 & 92.4 & 73.0 & 92.7 & 0.66 \\
\hline NASBA and p16 & & 48.4 & 97.4 & 83.9 & 87.2 & 0.46 \\
\hline FLOW and p16 & & 55.3 & 97.2 & 84.8 & 88.7 & 0.53 \\
\hline
\end{tabular}

Statistical measures have been calculated using all cases of the dataset (Table 1). Histology endpoint is CIN2+ for all cases. Definition of positivity of each medical test is presented in Table 15. CIN2+: cervical intraepithelial neoplasia grade 2 or worse, HR-HPV: high-risk human papillomavirus, NASBA: nucleic acid sequence based amplification for the identification of E6/E7 mRNA of the HPV types 16, 18, 31, 33, and 45, FLOW: flow cytometric E6/E7 HPV mRNA assay, PPV: positive predictive value, and NPV: negative predictive value.

most balanced results in terms of specificity, sensitivity, PPV, and NPV in comparison to the medical tests involved in this study and their combinations. The cutoff of CIN2+ was used because it is the decision threshold that a case is therapeutically handled; cases below CIN2+ are strictly monitored.

In our material, regarding the underestimated cases (CIN2+ cases which were classified by the CDSS as negatives or CIN1), only 1 out of the 4 misclassified ASCUS cases (Tables 12 and 13) was CIN3 and from the 13 misclassified non-ASCUS cases (Tables 7-9); 2 were CIN3 and 1 Adeno$\mathrm{Ca}$. Especially for the one misclassified adenocarcinoma, the total of the biomarkers was negative and the case was given as LSIL in cytology. On the other hand, the case of SCC that was given as LSIL in cytology (Table 4) was correctly classified by the PNN due to the fact that there were positive biomarkers (Table 7). Moreover, it is noteworthy that the CDSS classified correctly 9 of the 13 ASCUS cases and 17 of the 27 LSIL cases harbouring CIN2/3 (as presented in the results section).

In this study, the sensitivity of cytology using ASCUS+ as a cutoff was higher than HR-HPV DNA test (see Table 16: 98.1\% versus $89.4 \%$ ) in contrast to other studies, such as the ATHENA study [74]. The reader should be aware that this may be caused by verification bias related to the fact that cytology positive and HPV negative women had biopsies, in contrary to cytology negative and HPV positive women with a negative colposcopy. According to other studies [74] the sensitivity of HPV DNA test is higher than the sensitivity of cytology; however, in our case a special small population for referral to colposcopy is involved, in contrast to the generic population used in the ATHENA study. In addition, the laboratory bases the cytological examination on experienced cytopathologists as reported in our previous study [56] and thus the performance of the cytological examination is higher than the standard reported performance. In another study [75], the sensitivities of LBC and HPV DNA test are comparable with our results; additionally in another study [76] lower sensitivity of the HPV DNA test than the sensitivity of the cytological examination is reported. To conclude, performing our study on meta-analysis data would be impossible as detailed information for each individual case tests' results is required in order to train and test the CDSS system; therefore, a rather small but controlled population was preferred.

A potential application of this system is to support the decision of referring a woman to colposcopy or not. A work flow scenario is as follows: the cytological examination is used as primary test and only an ASCUS+ result is followed by the application of the other four ancillary tests using the remaining material in the vial. Subsequently, the five tests' results (including cytology) serve as inputs to the CDSS for evaluation and the CDSS outcome supports the final decision making for referring to colposcopy or not. The application of all five tests in general population would be a very costly 
TABLE 20: Performance of several type “AND” combinations between more than two test in detecting CIN2+.

\begin{tabular}{|c|c|c|c|c|c|c|}
\hline Combinations of medical tests & Cytology cutoff & Sensitivity (\%) & Specificity (\%) & PPV (\%) & NPV (\%) & Youden's index \\
\hline $\begin{array}{l}\text { Pap test and HPV DNA and } \\
\text { NASBA }\end{array}$ & ASCUS+ & 74.5 & 91.5 & 71.0 & 92.8 & 0.66 \\
\hline $\begin{array}{l}\text { Pap test and HPV DNA and } \\
\text { NASBA }\end{array}$ & LSIL+ & 70.2 & 92.9 & 73.4 & 91.8 & 0.63 \\
\hline $\begin{array}{l}\text { Pap test and HPV DNA and } \\
\text { NASBA }\end{array}$ & HSIL+ & 57.8 & 98.1 & 89.4 & 89.3 & 0.56 \\
\hline $\begin{array}{l}\text { Pap test and HPV DNA and } \\
\text { FLOW }\end{array}$ & ASCUS+ & 86.3 & 87.7 & 66.2 & 95.8 & 0.74 \\
\hline $\begin{array}{l}\text { Pap test and HPV DNA and } \\
\text { FLOW }\end{array}$ & LSIL+ & 80.7 & 88.9 & 67.0 & 94.3 & 0.70 \\
\hline $\begin{array}{l}\text { Pap test and HPV DNA and } \\
\text { FLOW }\end{array}$ & HSIL+ & 63.4 & 97.4 & 87.2 & 90.5 & 0.61 \\
\hline Pap test and HPV DNA and p16 & ASCUS+ & 55.3 & 94.8 & 74.8 & 88.4 & 0.50 \\
\hline Pap test and HPV DNA and p16 & LSIL+ & 53.4 & 95.0 & 74.8 & 88.0 & 0.48 \\
\hline Pap test and HPV DNA and p16 & HSIL+ & 46.6 & 98.6 & 90.4 & 86.9 & 0.45 \\
\hline $\begin{array}{l}\text { Pap test and HPV DNA and } \\
\text { NASBA and FLOW }\end{array}$ & ASCUS+ & 71.4 & 93.1 & 74.2 & 92.1 & 0.65 \\
\hline $\begin{array}{l}\text { Pap test and HPV DNA and } \\
\text { NASBA and FLOW }\end{array}$ & LSIL+ & 67.1 & 94.0 & 75.5 & 91.1 & 0.61 \\
\hline $\begin{array}{l}\text { Pap test and HPV DNA and } \\
\text { NASBA and FLOW }\end{array}$ & HSIL+ & 55.3 & 98.4 & 90.8 & 88.8 & 0.54 \\
\hline $\begin{array}{l}\text { Pap test and HPV DNA and } \\
\text { NASBA and FLOW and p16 }\end{array}$ & ASCUS+ & 44.7 & 98.8 & 91.1 & 86.5 & 0.44 \\
\hline $\begin{array}{l}\text { Pap test and HPV DNA and } \\
\text { NASBA and FLOW and p16 }\end{array}$ & LSIL+ & 43.5 & 98.8 & 90.9 & 86.3 & 0.42 \\
\hline $\begin{array}{l}\text { Pap test and HPV DNA and } \\
\text { NASBA and FLOW and p16 }\end{array}$ & HSIL+ & 38.5 & 99.5 & 95.4 & 85.3 & 0.38 \\
\hline $\begin{array}{l}\text { HPV DNA and NASBA and } \\
\text { FLOW }\end{array}$ & & 72.0 & 93.1 & 74.4 & 92.3 & 0.65 \\
\hline HPV DNA and NASBA and p16 & & 46.6 & 97.6 & 84.3 & 86.8 & 0.44 \\
\hline $\begin{array}{l}\text { HPV DNA and NASBA and } \\
\text { FLOW and p16 }\end{array}$ & & 45.3 & 98.8 & 91.3 & 86.7 & 0.44 \\
\hline NASBA and FLOW and p16 & & 47.2 & 98.8 & 91.6 & 87.1 & 0.46 \\
\hline
\end{tabular}

Statistical measures have been calculated using all cases of the dataset (Table 1). Histology endpoint is CIN2+ for all cases. Definition of positivity of each medical test is presented in Table 15. CIN2+: cervical intraepithelial neoplasia grade 2 or worse, HR-HPV: high-risk human papillomavirus, NASBA: nucleic acid sequence based amplification for the identification of E6/E7 mRNA of the HPV types 16, 18, 31, 33, and 45, FLOW: flow cytometric E6/E7 HPV mRNA assay, PPV: positive predictive value, and NPV: negative predictive value.

process and thus our method nowadays has the potential for application in the triage of ASCUS+ cases. However, a detailed cost/benefit, cost/effectiveness analysis is required as the cost of the tests is not the only factor that should be taken into account. Other important factors are the cost of the woman's transportation to a colposcopy clinic, especially in mountainous places, islands, or isolated cities/villages, the increment of recall time, and the psychological effects to the woman and her family among others.
In the literature there are already simpler techniques proposed for the triage of ASCUS and LSIL, such as the repeat cytology and the application of mRNA testing. As mentioned in [77-79], the use of NASBA HPV mRNA test in triage of women with ASCUS and LSIL may reduce the referral rate to colposcopy. As presented in [77], the HPV mRNA test significantly reduced the time from the first abnormal cytology until biopsy and had predictive values comparable with those of repeat cytology. In [79], the authors report that 
HPV mRNA testing is a better triage test for women with LSIL than repeat cytology, as it was more sensitive (94.2\%) and specific $(86.0 \%)$ for detecting CIN2+. In addition, the HPV mRNA test showed higher PPV (67.0\%) compared to repeat cytology (38.4\%). In a meta-analysis of the accuracy of mRNA testing for detecting CIN2+, the mRNA testing was substantially more specific than the HPV DNA test in women with ASCUS and LSIL [79]. However, it demonstrated lower sensitivity and thus women with negative mRNA test results cannot be considered free of $\mathrm{CIN} 2+$ and require followup [79]. In our study, the proposed system showed higher sensitivity, higher specificity, higher PPV, and higher NPV compared to NASBA mRNA testing, for detecting CIN2+. In comparison to the HPV DNA test, the proposed system is a little less sensitive in detecting CIN2+; however, its specificity and PPV are significantly higher. According to our results, the proposed system produced the most balanced results in terms of sensitivity, specificity, PPV, and NPV and demonstrated the highest Youden's index, compared to cytology and the biomarkers used in the study and their combinations. Thus, in comparison to the already proposed schemes for triage of ASCUS+, our approach may produce more accurate results, leading to improved triage of ASCUS+ and improved detection of CIN2+. Therefore, the overhead for both cytological laboratories and colposcopy rooms can be reduced.

The application of the proposed CDSS gave promising results, suggesting that such an approach may significantly improve the accuracy of diagnosis. Furthermore, the notable performance of the CDSS in identifying women with LSIL cytology at risk of developing cancer suggests that such systems may play an important role in triage decisions and hence may reduce the overload of colposcopy clinics and guide personalised management and therapeutic interventions. The results should be further assessed in larger datasets in order to confirm the reproducibility of these findings. As some of the tests and biomarkers may result in increased cost, our research is now directed to develop a more cost-effective CDSS which will use fewer tests, without losing much in performance. Furthermore, machine learning techniques for handling missing values are under examination, in order to be able to provide outcomes also for cases with missing or invalid examinations' results.

Today, the CDSS is available to users as a PC application. Our future work involves the upgrade of the CDSS to an intelligent web service for patient-specific prediction, progression, and prognosis of cervical cancer, available over the Internet to the worldwide medical community, which will serve as a decision support system to physicians and medical researchers for the management of new cases or the followup of existing cases.

\section{Conflict of Interests}

The authors declare that there is no conflict of interests regarding the publication of this paper.

\section{Acknowledgments}

This study was funded by the Greek Ministry of Development (General Secretariat for Research and TechnologyGSRT), Project "HPVGuard", Cooperation 2011-2013 (Code: $11 \Sigma Y$ N_10_250). A part of the data is inherited from the Project "AKAKOS" (GSRT Code: ATT 95) and the project "study and evaluation of the methods, for the prognosis of success for vaccination against HPV infections-Ygeia Proneia 2000-2006" (Greek Ministry of Health). Finally, the authors would like to thank the reviewers for their comments that helped to improve this paper.

\section{References}

[1] A. Jemal, F. Bray, M. M. Center, J. Ferlay, E. Ward, and D. Forman, "Global cancer statistics," CA: A Cancer Journal for Clinicians, vol. 61, no. 2, pp. 69-90, 2011.

[2] J. M. Walboomers, M. V. Jacobs, M. M. Manos et al., "Human papillomavirus is a necessary cause of invasive cervical cancer worldwide," The Journal of Pathology, vol. 189, no. 1, pp. 12-19, 1999.

[3] E. F. Dunne, E. R. Unger, M. Sternberg et al., "Prevalence of HPV infection among females in the United States," The Journal of the American Medical Association, vol. 297, no. 8, pp. 813-819, 2007.

[4] N. Munoz, F. X. Bosch, S. de Sanjose et al., "Epidemiologic classification of human papillomavirus types associated with cervical cancer," The New England Journal of Medicine, vol. 348, no. 6, pp. 518-527, 2003.

[5] M. H. Mayrand, E. Duarte-Franco, I. Rodrigues et al., "Human papillomavirus DNA versus Papanicolaou screening tests for cervical cancer," The New England Journal of Medicine, vol. 357, no. 16, pp. 1579-1588, 2007.

[6] A. Mathew and P. S. George, "Trends in incidence and mortality rates of squamous cell carcinoma and adenocarcinoma of cervix-worldwide," Asian Pacific Journal of Cancer Prevention, vol. 10, no. 4, pp. 645-650, 2009.

[7] J. Cuzick, M. Arbyn, R. Sankaranarayanan et al., "Overview of human papillomavirus-based and other novel options for cervical cancer screening in developed and developing countries," Vaccine, vol. 26, supplement 10, pp. K29-K41, 2008.

[8] P. Naucler, W. Ryd, S. Tornberg et al., "Efficacy of HPV DNA testing with cytology triage and/or repeat HPV DNA testing in primary cervical cancer screening," Journal of the National Cancer Institute, vol. 101, no. 2, pp. 88-99, 2009.

[9] M. Arbyn, P. Sasieni, C. J. Meijer, C. Clavel, G. Koliopoulos, and J. Dillner, "Chapter 9: clinical applications of HPV testing: a summary of meta-analyses," Vaccine, vol. 24, supplement 3, pp. S78-S89, 2006.

[10] J. Cuzick, A. Szarewski, H. Cubie et al., "Management of women who test positive for high-risk types of human papillomavirus: the HART study," The Lancet, vol. 362, no. 9399, pp. 1871-1876, 2003.

[11] H. L. Smits, B. van Gemen, R. Schukkink et al., "Application of the NASBA nucleic acid amplification method for the detection of human papillomavirus type 16 E6-E7 transcripts," Journal of Virological Methods, vol. 54, no. 1, pp. 75-81, 1995.

[12] J. Mockel, J. Quaas, H. Meisel, A. S. Endres, and V. Schneider, "Human papillomavirus E6/E7 mRNA testing has higher specificity than liquid-based DNA testing in the evaluation of 
cervical intraepithelial neoplasia," Analytical and Quantitative Cytology and Histology, vol. 33, no. 6, pp. 311-315, 2011.

[13] A. Szarewski, D. Mesher, L. Cadman et al., "Comparison of seven tests for high-grade cervical intraepithelial neoplasia in women with abnormal smears: the Predictors 2 study," Journal of Clinical Microbiology, vol. 50, no. 6, pp. 1867-1873, 2012.

[14] C. Kottaridi, S. Tsiodras, A. Spathis et al., "Clinical performance of human papillomavirus E6, E7 mRNA flow cytometric assay compared to human papillomavirus DNA typing," Analytical and Quantitative Cytology and Histology, vol. 33, no. 6, pp. 305310, 2011.

[15] A. Trope, K. Sjoborg, A. Eskild et al., "Performance of human papillomavirus DNA and mRNA testing strategies for women with and without cervical neoplasia," Journal of Clinical Microbiology, vol. 47, no. 8, pp. 2458-2464, 2009.

[16] S. W. Sorbye, S. Fismen, T. Gutteberg, and E. S. Mortensen, "Triage of women with minor cervical lesions: data suggesting a "test and treat" approach for HPV E6/E7 mRNA testing," PLoS ONE, vol. 5, no. 9, article e12724, 2010.

[17] R. Narimatsu and B. K. Patterson, "High-throughput cervical cancer screening using intracellular human papillomavirus E6 and E7 mRNA quantification by flow cytometry," American Journal of Clinical Pathology, vol. 123, no. 5, pp. 716-723, 2005.

[18] G. Coquillard, B. Palao, and B. K. Patterson, "Quantification of intracellular HPV E6/E7 mRNA expression increases the specificity and positive predictive value of cervical cancer screening compared to HPV DNA," Gynecologic Oncology, vol. 120, no. 1, pp. 89-93, 2011.

[19] G. Valasoulis, I. Tsoumpou, C. Founta et al., "The role of p16 ${ }^{\text {INK4a }}$ immunostaining in the risk assessment of women with LSIL cytology: a prospective pragmatic study," European Journal of Gynaecological Oncology, vol. 32, no. 2, pp. 150-152, 2011.

[20] I. Tsoumpou, M. Arbyn, M. Kyrgiou et al., "p16 ${ }^{\mathrm{INK} 4 \mathrm{a}}$ immunostaining in cytological and histological specimens from the uterine cervix: a systematic review and meta-analysis," Cancer Treatment Reviews, vol. 35, no. 3, pp. 210-220, 2009.

[21] M. Benevolo, A. Vocaturo, D. Caraceni et al., "Sensitivity, specificity, and clinical value of human papillomavirus (HPV) E6/E7 mRNA assay as a triage test for cervical cytology and HPV DNA test," Journal of Clinical Microbiology, vol. 49, no. 7, pp. 2643-2650, 2011.

[22] K. Cuschieri and N. Wentzensen, "Human papillomavirus mRNA and p16 detection as biomarkers for the improved diagnosis of cervical neoplasia," Cancer Epidemiology, Biomarkers \& Prevention, vol. 17, no. 10, pp. 2536-2545, 2008.

[23] F. M. Carozzi, "Combined analysis of HPV DNA and p16 ${ }^{\text {INK4a }}$ expression to predict prognosis in ASCUS and LSIL pap smears," Collegium Antropologicum, vol. 31, supplement 2, pp. 103-106, 2007.

[24] K. J. Denton, C. Bergeron, P. Klement, M. J. Trunk, T. Keller, and R. Ridder, "The sensitivity and specificity of p16 ${ }^{\text {INK4a }}$ cytology vs HPV testing for detecting high-grade cervical disease in the triage of ASC-US and LSIL pap cytology results," American Journal of Clinical Pathology, vol. 134, no. 1, pp. 12-21, 2010.

[25] K. Kawamoto, C. A. Houlihan, E. A. Balas, and D. F. Lobach, "Improving clinical practice using clinical decision support systems: a systematic review of trials to identify features critical to success," British Medical Journal, vol. 330, article 765, 2005.

[26] K. Kawamoto, D. F. Lobach, H. F. Willard, and G. S. Ginsburg, "A national clinical decision support infrastructure to enable the widespread and consistent practice of genomic and personalized medicine," BMC Medical Informatics and Decision Making, vol. 9, no. 1, article 17, 2009.

[27] P. J. Lisboa and A. F. G. Taktak, "The use of artificial neural networks in decision support in cancer: a systematic review," Neural Networks, vol. 19, no. 4, pp. 408-415, 2006.

[28] S. Y. Park, D. Sargent, R. Lieberman, and U. Gustafsson, "Domain-specific image analysis for cervical neoplasia detection based on conditional random fields," IEEE Transactions on Medical Imaging, vol. 30, no. 3, pp. 867-878, 2011.

[29] M. Jondet, R. Agoli-Agbo, and L. Dehennin, "Automatic measurement of epithelium differentiation and classification of cervical intraneoplasia by computerized image analysis," Diagnostic Pathology, vol. 5, no. 1, article 7, 2010.

[30] Y. Srinivasan, E. Corona, B. Nutter, S. Mitra, and S. Bhattacharya, "A unified model-based image analysis framework for automated detection of precancerous lesions in digitized uterine cervix images," IEEE Journal on Selected Topics in Signal Processing, vol. 3, no. 1, pp. 101-111, 2009.

[31] D. Ramli, A. Kadmin, M. Mashor, N. Ashidi, and M. Isa, "Diagnosis of cervical cancer using hybrid multilayered perceptron (HMLP) network," in Knowledge-Based Intelligent Information and Engineering Systems, M. Negoita, R. Howlett, and L. Jain, Eds., Lecture Notes in Computer Science, pp. 591-598, Springer, Berlin, Germany, 2004.

[32] C. E. Pedreira, L. Macrini, M. G. Land, and E. S. Costa, "New decision support tool for treatment intensity choice in childhood acute lymphoblastic leukemia," IEEE Transactions on Information Technology in Biomedicine, vol. 13, no. 3, pp. 284290, 2009.

[33] F. K. Ahmad, S. Deris, and M. S. Abdullah, "Synergy network based inference for breast cancer metastasis," Procedia Computer Science, vol. 3, pp. 1094-1100, 2011.

[34] J. M. Jerez-Aragones, J. A. Gomez-Ruiz, G. Ramos-Jimenez, J. Munoz-Perez, and E. Alba-Conejo, "A combined neural network and decision trees model for prognosis of breast cancer relapse," Artificial Intelligence in Medicine, vol. 27, no. 1, pp. 4563, 2003.

[35] H.-C. Lin, H.-C. Wu, C.-H. Chang, T.-C. Li, W.-M. Liang, and J.-Y. W. Wang, "Development of a real-time clinical decision support system upon the web mvc-based architecture for prostate cancer treatment," BMC Medical Informatics and Decision Making, vol. 11, no. 1, article 16, 2011.

[36] P. Karakitsos, C. Chrelias, A. Pouliakis, G. Koliopoulos, A. Spathis et al., "Identification of women for referral to colposcopy by neural networks: a preliminary study based on LBC and molecular biomarkers," Journal of Biomedicine and Biotechnology, vol. 2012, Article ID 303192, 8 pages, 2012.

[37] P. Karakitsos, A. Pouliakis, C. Meristoudis et al., "A preliminary study of the potential of tree classifiers in triage of high-grade squamous intraepithelial lesions," Analytical and Quantitative Cytology and Histology, vol. 33, no. 3, pp. 132-140, 2011.

[38] M. R. Henry, “The Bethesda System 2001: an update of new terminology for gynecologic cytology," Clinics in Laboratory Medicine, vol. 23, no. 3, pp. 585-603, 2003.

[39] J. H. Smith, "Bethesda 2001," Cytopathology, vol. 13, no. 1, pp. $4-10,2002$.

[40] J. J. Gomez-Roman, C. Echevarria, S. Salas et al., "A type-specific study of human papillomavirus prevalence in cervicovaginal samples in three different Spanish regions," Acta Pathologica Microbiologica et Immunologica Scandinavica, vol. 117, no. 1, pp. 22-27, 2009. 
[41] S. Tyagi, D. P. Bratu, and F. R. Kramer, "Multicolor molecular beacons for allele discrimination," Nature Biotechnology, vol. 16, no. 1, pp. 49-53, 1998.

[42] R. Klaes, A. Benner, T. Friedrich et al., "p16 ${ }^{\mathrm{INK} 4 \mathrm{a}}$ immunohistochemistry improves interobserver agreement in the diagnosis of cervical intraepithelial neoplasia," The American Journal of Surgical Pathology, vol. 26, no. 11, pp. 1389-1399, 2002.

[43] S. Theodoridis and K. Koutroumbas, Pattern Recognition, Elsevier, London, UK, 4th edition, 2009.

[44] P. Bountris, A. Apostolou, M. Haritou, E. Passalidou, and D. Koutsouris, "Combined texture features for improved classification of suspicious areas in autofluorescence bronchoscopy," in Proceedings of the 9th International Conference on Information Technology and Applications in Biomedicine (ITAB '09), pp. 1-4, Larnaca, Cyprus, November 2009.

[45] C. Ding and H. Peng, "Minimum redundancy feature selection from microarray gene expression data," in Proceedings of the IEEE Computational Systems Bioinformatics (CSB '03), pp. 523530, Stanford, Calif, USA, August 2003.

[46] D. Michie, D. J. Spiegelhalter, and C. C. Taylor, Machine Learning, Neural and Statistical Classification, Ellis Horwood, New York, NY, USA, 1994.

[47] R. O. Duda, P. E. Hart, and D. G. Stork, Pattern Classification, Wiley, New York, NY, USA, 2nd edition, 2001.

[48] L. Breiman, Classification and Regression Trees, Wadsworth International Group, Belmont, Calif, USA, 1984.

[49] D. F. Specht, "Probabilistic neural networks and the polynomial Adaline as complementary techniques for classification," IEEE Transactions on Neural Networks, vol. 1, no. 1, pp. 111-121, 1990.

[50] P. D. Wasserman, Advanced Methods in Neural Computing, Van Nostrand Reinhold, New York, NY, USA, 1993.

[51] Y. Bengio and Y. LeCun, "Scaling learning algorithms towards AI," in Large-Scale Kernel Machines, L. Bottou, O. Chapelle, D. DeCoste, and J. Weston, Eds., pp. 321-360, The MIT Press, Cambridge, Mass, USA, 2007.

[52] U. Braga-Neto and E. Dougherty, "Exact performance of error estimators for discrete classifiers," Pattern Recognition, vol. 38, no. 11, pp. 1799-1814, 2005.

[53] U. M. Braga-Neto, "Classification and error estimation for discrete data," Current Genomics, vol. 10, no. 7, pp. 446-462, 2009.

[54] H. F. Nauth, Gynecological Cytology, Thieme, Stuttgart, Germany, 2007.

[55] G. Koliopoulos, C. Chrelias, A. Pappas, S. Makridima, E. Kountouris et al., "The diagnostic accuracy of two methods for E6\&7 mRNA detection in women with minor cytological abnormalities," Acta Obstetricia et Gynecologica Scandinavica, vol. 91, no. 7, pp. 794-801, 2012.

[56] A. Spathis, C. Kottaridi, A. Chranioti, C. Meristoudis, C. Chrelias et al., "mRNA and DNA detection of human papillomaviruses in women of all ages attending two colposcopy clinics," PLoS ONE, vol. 7, no. 11, article e49205, 2012.

[57] T. Kelesidis, L. Aish, M. A. Steller et al., "Human papillomavirus (HPV) detection using in situ hybridization in histologic samples," American Journal of Clinical Pathology, vol. 136, no. 1, pp. 119-127, 2011.

[58] M. Nasioutziki, A. Daniilidis, K. Dinas et al., "The evaluation of $\mathrm{p} 16^{\mathrm{INK} 4 \mathrm{a}}$ immunoexpression/immunostaining and human papillomavirus DNA test in cervical liquid-based cytological samples," International Journal of Gynecological Cancer, vol. 21, no. 1, pp. 79-85, 2011.
[59] A. Spathis, E. Aga, M. Alepaki et al., "Promoter methylation of p16 ${ }^{\mathrm{INK} 4 \mathrm{~A}}$, hMLH1, and MGMT in liquid-based cervical cytology samples compared with clinicopathological findings and HPV presence," Infectious Diseases in Obstetrics and Gynecology, vol. 2011, Article ID 927861, 5 pages, 2011.

[60] I. Tsoumpou, G. Valasoulis, C. Founta et al., "High-risk human papillomavirus DNA test and p16 ${ }^{\mathrm{INK} 4 \mathrm{a}}$ in the triage of LSIL: a prospective diagnostic study," Gynecologic Oncology, vol. 121, no. 1, pp. 49-53, 2011.

[61] G. Valasoulis, G. Koliopoulos, C. Founta et al., "Alterations in human papillomavirus-related biomarkers after treatment of cervical intraepithelial neoplasia," Gynecologic Oncology, vol. 121, no. 1, pp. 43-48, 2011.

[62] J. Roelens, M. Reuschenbach, M. von Knebel Doeberitz, N. Wentzensen, C. Bergeron, and M. Arbyn, "p16 ${ }^{\mathrm{INK} 4 \mathrm{a}}$ immunocytochemistry versus human papillomavirus testing for triage of women with minor cytologic abnormalities: a systematic review and meta-analysis," Cancer Cytopathology, vol. 120, no. 5, pp. 294-307, 2012.

[63] M. Arbyn, P. Martin-Hirsch, F. Buntinx, M. van Ranst, E. Paraskevaidis, and J. Dillner, "Triage of women with equivocal or low-grade cervical cytology results: a meta-analysis of the HPV test positivity rate," Journal of Cellular and Molecular Medicine, vol. 13, no. 4, pp. 648-659, 2009.

[64] G. Koliopoulos, M. Arbyn, P. Martin-Hirsch, M. Kyrgiou, W. Prendiville, and E. Paraskevaidis, "Diagnostic accuracy of human papillomavirus testing in primary cervical screening: a systematic review and meta-analysis of non-randomized studies," Gynecologic Oncology, vol. 104, no. 1, pp. 232-246, 2007.

[65] M. Arbyn, F. Buntinx, M. van Ranst, E. Paraskevaidis, P. MartinHirsch, and J. Dillner, "Virologic versus cytologic triage of women with equivocal pap smears: a meta-analysis of the accuracy to detect high-grade intraepithelial neoplasia," Journal of the National Cancer Institute, vol. 96, no. 4, pp. 280-293, 2004.

[66] R. S. Hoda and S. A. Hoda, Fundamentals of PAP Test Cytology, Humana Press, Totowa, NJ, USA, 2007.

[67] London Quality Assurance Reference Centre, "London Annual Report 2005-2006," Tech. Rep. KC61, London Quality Assurance Reference Centre, London, UK, 2006.

[68] B. A. Jones and D. A. Novis, "Cervical biopsy-cytology correlation. A College of American Pathologists Q-Probes study of 22 439 correlations in 348 laboratories," Archives of Pathology and Laboratory Medicine, vol. 120, no. 6, pp. 523-531, 1996.

[69] M. Kyrgiou, I. Tsoumpou, T. Vrekoussis et al., "The up-todate evidence on colposcopy practice and treatment of cervical intraepithelial neoplasia: the cochrane colposcopy \& cervical cytopathology collaborative group (C5 group) approach," Cancer Treatment Reviews, vol. 32, no. 7, pp. 516-523, 2006.

[70] M. Arbyn, M. Kyrgiou, C. Simoens et al., "Perinatal mortality and other severe adverse pregnancy outcomes associated with treatment of cervical intraepithelial neoplasia: meta-analysis," British Medical Journal, vol. 337, Article ID a1284, 2008.

[71] E. Paraskevaidis, M. Kyrgiou, and P. Martin-Hirsch, "Have we dismissed ablative treatment too soon in colposcopy practice?" BJOG, vol. 114, no. 1, pp. 3-4, 2007.

[72] M. Kyrgiou, G. Koliopoulos, P. Martin-Hirsch, M. Arbyn, W. Prendiville, and E. Paraskevaidis, "Obstetric outcomes after conservative treatment for intraepithelial or early invasive cervical lesions: systematic review and meta-analysis," The Lancet, vol. 367, no. 9509, pp. 489-498, 2006.

[73] M. Arbyn, E. Paraskevaidis, P. Martin-Hirsch, W. Prendiville, and J. Dillner, "Clinical utility of HPV-DNA detection: triage 
of minor cervical lesions, follow-up of women treated for high-grade CIN: an update of pooled evidence," Gynecologic Oncology, vol. 99, no. 3, supplement, pp. S7-S11, 2005.

[74] P. E. Castle, M. H. Stoler, T. C. Wright Jr., A. Sharma, T. L. Wright, and C. M. Behrens, "Performance of carcinogenic human papillomavirus (HPV) testing and HPV16 or HPV18 genotyping for cervical cancer screening of women aged 25 years and older: a subanalysis of the ATHENA study," The Lancet Oncology, vol. 12, no. 9, pp. 880-890, 2011.

[75] C. Ferreccio, M. C. Bratti, M. E. Sherman et al., "A comparison of single and combined visual, cytologic, and virologic tests as screening strategies in a region at high risk of cervical cancer," Cancer Epidemiology, Biomarkers \& Prevention, vol. 12, no. 9, pp. 815-823, 2003.

[76] M. Schiffman, R. Herrero, A. Hildesheim et al., "HPV DNA testing in cervical cancer screening: results from women in a high risk province of Costa Rica," The Journal of the American Medical Association, vol. 283, no. 1, pp. 87-93, 2000.

[77] S. W. Sorbye, S. Fismen, T. J. Gutteberg, E. S. Mortensen, and F. E. Skjeldestad, "HPV mRNA testing in triage of women with ASC-US cytology may reduce the time for CIN2+diagnosis compared with repeat cytology," Current Pharmaceutical Design, vol. 19, no. 8, pp. 1401-1405, 2013.

[78] F. Verdoodt, A. Szarewski, P. Halfon, K. Cuschieri, and M. Arbyn, "Triage of women with minor abnormal cervical cytology: meta-analysis of the accuracy of an assay targeting messenger ribonucleic acid of 5 high-risk human papillomavirus types," Cancer Cytopathology, vol. 121, no. 12, pp. 675-687, 2013.

[79] S. W. Sorbye, M. Arbyn, S. Fismen, T. J. Gutteberg, and E. S. Mortensen, "Triage of women with low-grade cervical lesionsHPV mRNA testing versus repeat cytology," PLoS ONE, vol. 6, no. 8, article e24083, 2011. 

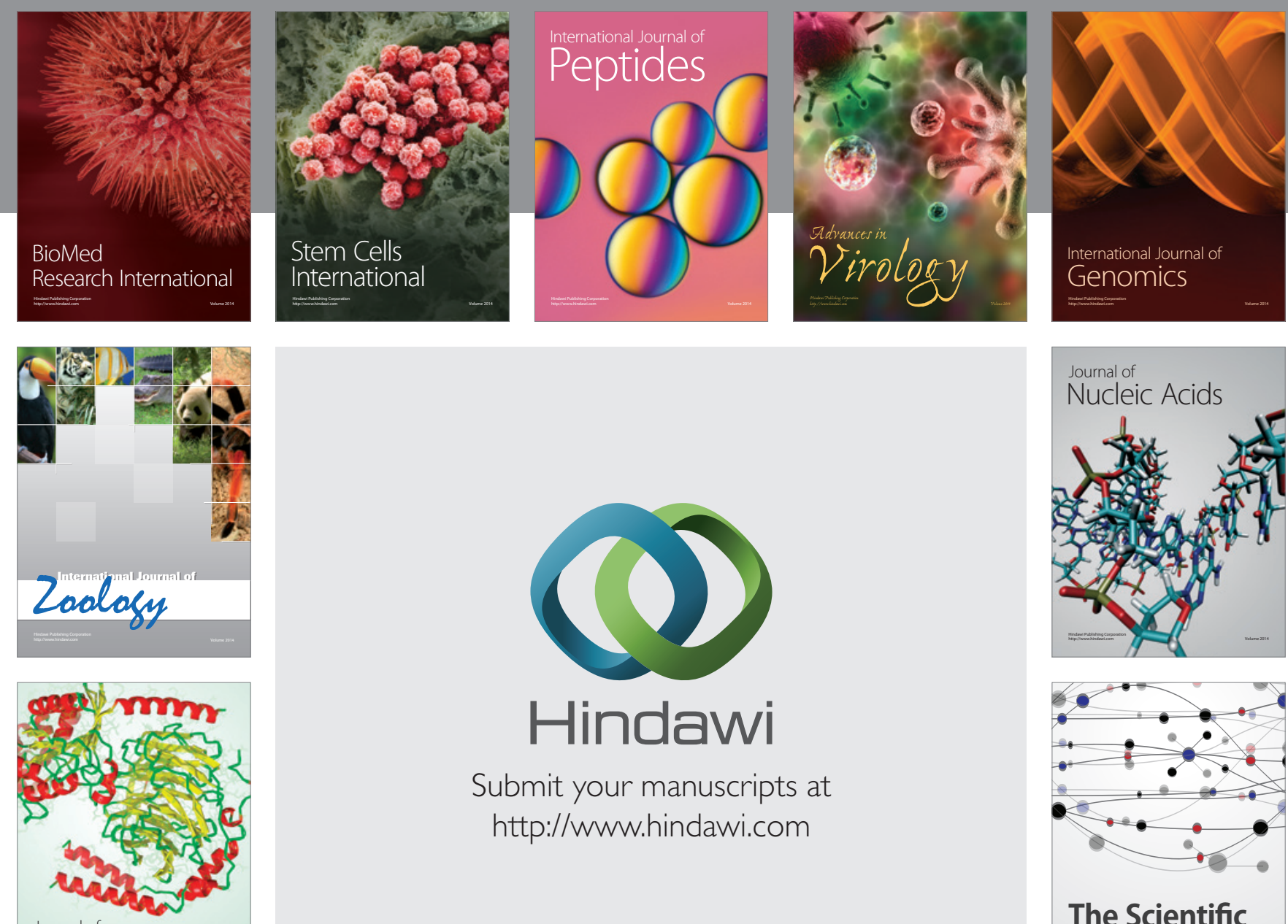

Submit your manuscripts at

http://www.hindawi.com

Journal of
Signal Transduction
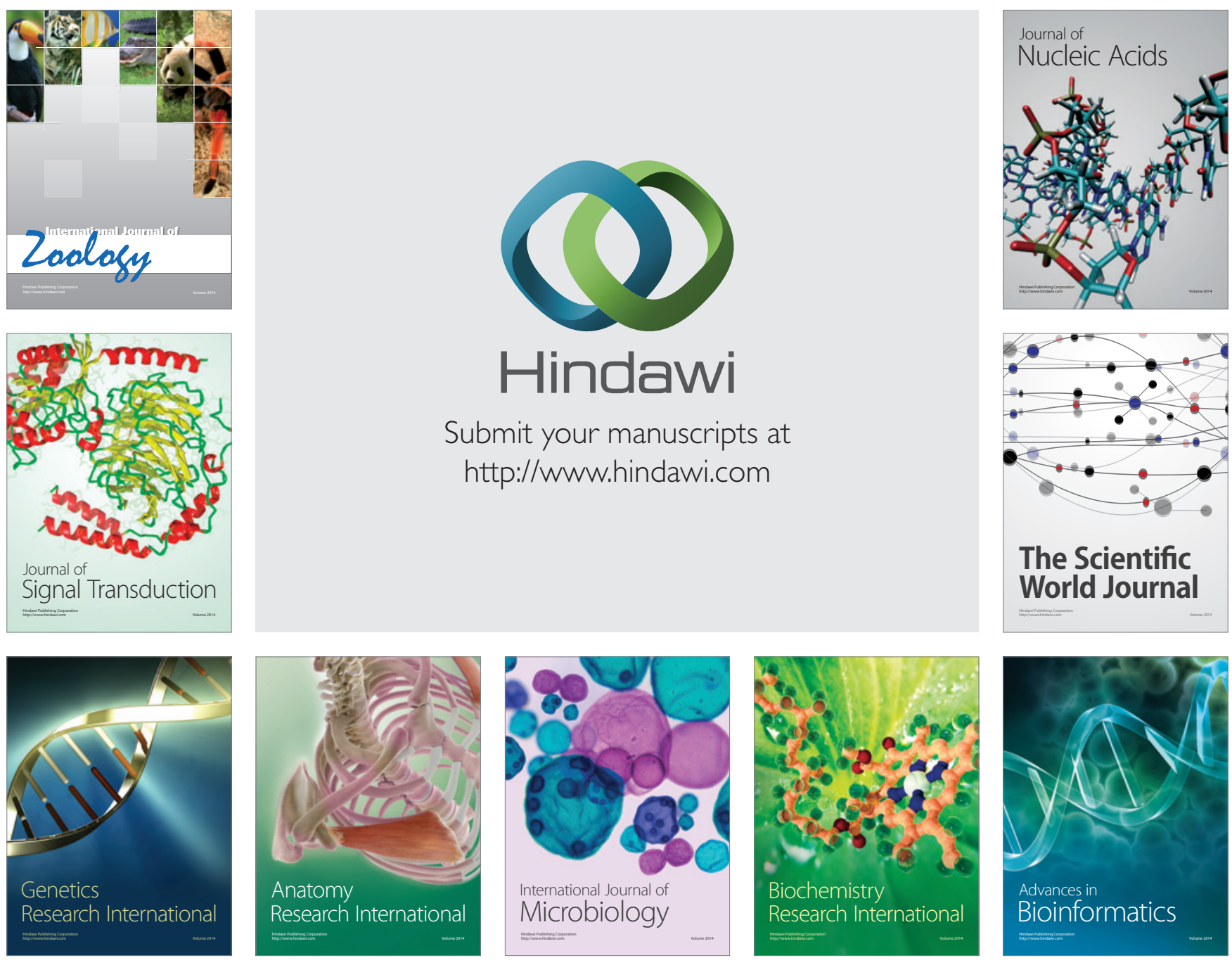

The Scientific World Journal
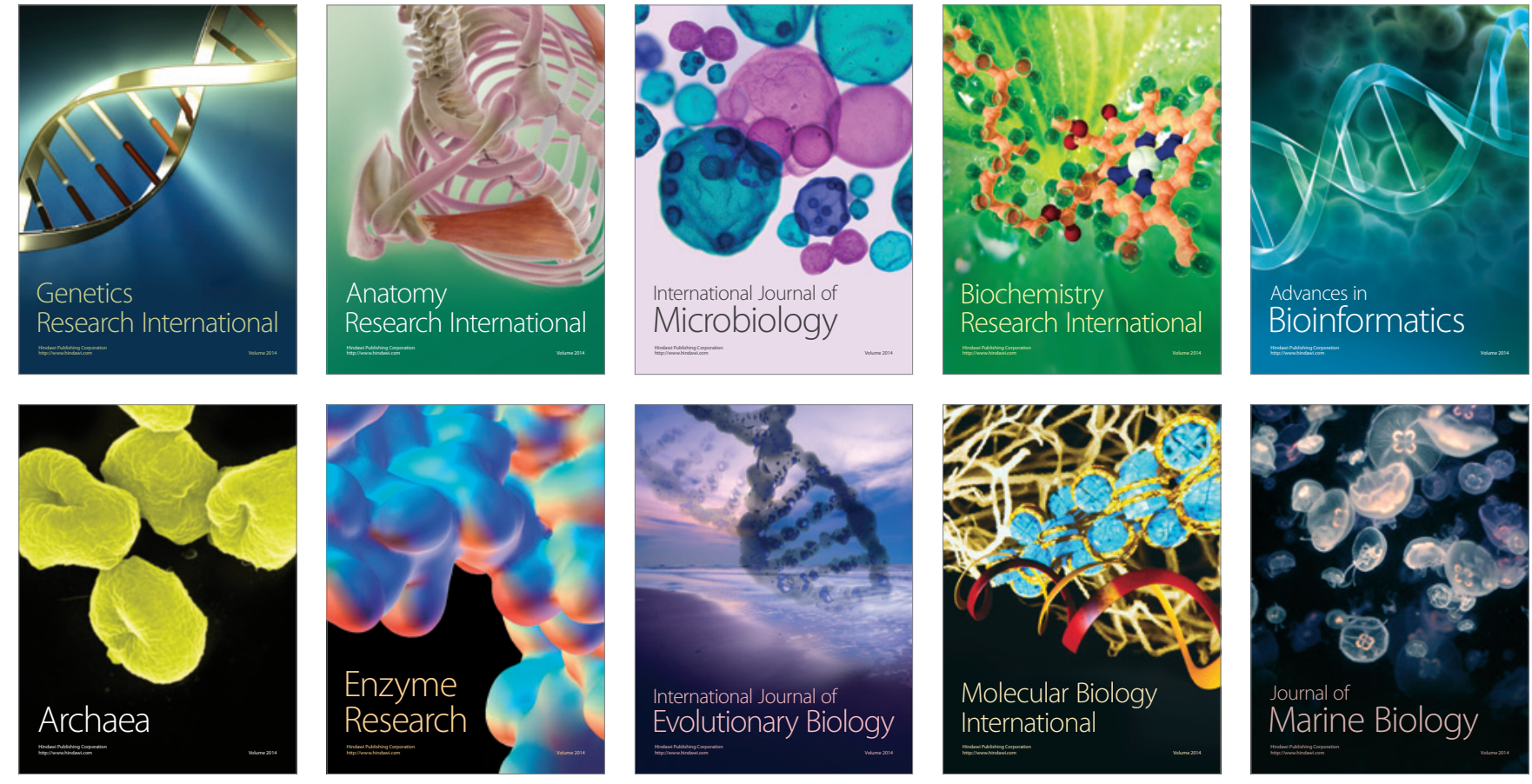